\title{
Spherical Resonator for Vapor-Phase Speed of Sound and Measurements of 1,1,1,2,2,3,3-heptafluoro-3-methoxypropane (RE347mcc) and trans-1,3,3,3-tetrafluoropropene $[\mathrm{R} 1234 \mathrm{ze}(\mathrm{E})]^{1}$
}

\author{
Richard A. Perkins ${ }^{2}$, Mark O. McLinden \\ Applied Chemicals and Materials Division, National Institute of Standards and Technology \\ 325 Broadway, Mailstop 647.07, Boulder, Colorado 80305 USA \\ Version of 14 May 2015 \\ to be submitted to J. Chem. Thermodynamics
}

\begin{abstract}
We describe an apparatus to measure the speed of sound of gas samples at temperatures from $265 \mathrm{~K}$ to $500 \mathrm{~K}$ with pressures up to $10 \mathrm{MPa}$. The speed of sound was determined from the frequency of the three lowest-order radial resonance modes for the gas in a spherical cavity machined from type 321 stainless steel for corrosion resistance. The spherical resonator was contained in an isothermal copper block that was maintained at the temperature of interest by a multilayer thermostat with vacuum insulation. The dimensions of the spherical cavity were characterized as a function of temperature and pressure though calibration measurements with high-purity argon. The performance of the apparatus was demonstrated with measurements of high-purity methane and ethane. Measurements of the sound speed of 1,1,1,2,2,3,3-heptafluoro3-methoxypropane (RE347mcc) are reported at temperatures from $325 \mathrm{~K}$ to $500 \mathrm{~K}$ with pressures up to 1.6 $\mathrm{MPa}$. Measurements on trans-1,3,3,3-tetrafluoropropene (R1234ze(E)) are reported at temperatures from $280 \mathrm{~K}$ to $420 \mathrm{~K}$ with pressures up to $2.8 \mathrm{MPa}$. The average combined expanded uncertainties of the measured sound speed for RE347mcc and R1234ze(E) are $0.018 \%$ and $0.041 \%$, respectively.
\end{abstract}

\section{Keywords}

ethane; 1,1,1,2,2,3,3-heptafluoro-3-methoxypropane; methane; sound speed; spherical resonator; trans-1,3,3,3-tetrafluoropropene

\footnotetext{
${ }^{1}$ Contribution of the National Institute of Standards and Technology. Not subject to copyright in the United States.

${ }^{2}$ Corresponding author. Tel.: +1 303497 5499; fax: +1 3034976682 .

Email addresses: richard.perkins@nist.gov (R. A. Perkins), mark.mclinden@ nist.gov (M. O.

McLinden).
} 


\section{Introduction}

We describe an apparatus to measure the sound speed of gas-phase samples at temperatures up to $500 \mathrm{~K}$ and pressures up to $10 \mathrm{MPa}$. The speed of sound is determined from the frequency of the three lowest-order radial resonance modes for the gas in a spherical cavity. The dimensions of the spherical cavity are characterized through calibration measurements with high-purity argon gas as a function of temperature and pressure (up to $10 \mathrm{MPa}$ ). The performance of the apparatus is demonstrated with measurements of high-purity methane and ethane.

Our instrument is intended for fluid property measurements on chemicals of industrial interest. The resonator comprises two flanged hemispheres (type 321 stainless steel) that are bolted together, and which serve as the pressure vessel to contain the sample in a spherical cavity. This is in contrast to some other instruments in which bolted hemispheres are contained within a separate pressure vessel $[1,2]$ or a welded sphere serves as a pressure vessel.[3] Our design allows for disassembly of the sphere for inspection and cleaning; it also requires less sample than would be required if the sphere were housed within a separate pressure vessel. These are significant advantages when working with samples that might be expensive or hazardous or that might decompose during testing. The more complex geometry of the flanged design does, however, complicate the analysis of various corrections to the resonance frequencies, and this increases the uncertainty of the measurements. The resulting data are suitable for the development of equations of state.

Measurements of the speed of sound of 1,1,1,2,2,3,3-heptafluoro-3-methoxypropane (RE347mcc) are reported at temperatures from (325 to 500) $\mathrm{K}$, with pressures up to $1.6 \mathrm{MPa}$. RE347mcc, also known by the trade names HFE-7000 and Novec ${ }^{\mathrm{TM}} 7000,{ }^{3}$ is of interest as a refrigerant, as the working fluid in organic Rankine-cycle power systems, and as a lowtemperature heat-transfer fluid. It has a global warming potential (100 year time horizon, $\mathrm{GWP}_{100}$ ) of 530 [4], zero ozone depletion potential (ODP), and an atmospheric lifetime of 5.0 years. It is nonflammable and of low acute toxicity with an occupational exposure limit (OEL) of 75 ppm, and a "no observed adverse effect level" (NOAEL) of 1000 ppm [5].

Measurements are also reported on trans-1,3,3,3-tetrafluoropropene (R1234ze(E)) at temperatures from $280 \mathrm{~K}$ to $420 \mathrm{~K}$ with pressures up to $2.8 \mathrm{MPa}$. R1234ze(E) is an unsaturated hydroflouro-olefin (HFO) that has been identified as a promising working fluid for refrigeration cycles. R1234ze(E) has zero ODP and an extremely short atmospheric lifetime (4.9 days) due to

\footnotetext{
${ }^{3}$ Certain trade names and products are given to adequately document the experimental equipment and procedures. This does not constitute a recommendation or endorsement of these products by the National Institute of Standards and Technology, nor does it imply that the products are necessarily the best available for the purpose.
} 
reactivity with atmospheric hydroxyl radicals, resulting in a $\mathrm{GWP}_{100}<1$ [4]. This fluid is of low toxicity, but it is very slightly flammable; it has been assigned a safety classification of A2L under ANSI/ASHRAE Standard 34 [6].

\section{Experimental}

\subsection{Measuring principle}

The spherical acoustic resonator is an accurate and reliable tool to measure the sound speed of gases. The behavior of spherical acoustic resonators has been thoroughly modeled and experimentally verified. Specialized spherical resonators have been used to determine the gas constant and as acoustic thermometry standards with very low uncertainty. Excellent descriptions of spherical acoustic resonators and their theory of operation have been reported by Moldover et al. [2], Trusler [7] and Moldover et al.[1]

The working equation for spherical acoustic resonators relates the sound speed $w$ to eigenvalues $v_{l n}$, sphere radius $r$, and measured resonance frequency $f_{l n}$

$$
f_{l n}+i g_{l n}=v_{l n}\left(\frac{w}{2 \pi r}\right)+\sum_{j}(\Delta f+i g)_{j}
$$

where $g_{l n}$ is the half-width and the summation over $j$ accounts for small corrections to the ideal model. The eigenvalues $v_{l n}$ are given by the $n$ turning points, where the derivatives of the spherical Bessel function of order $l, j_{l}(x)$, are equal to zero $\left(\mathrm{d} j_{l}(x) / \mathrm{d} x\right)=0$. The radial resonance modes $(l=0)$ are preferred, although the first-order $(l=1)$ modes can sometimes be useful for fluids with large sound absorption.[8]

The most significant corrections account for the thermal and viscous boundary layers at low pressures, coupling between gas and shell motion, fluid dissipation (bulk viscosity), and perturbation of the spherical resonance by the filling tubes and by the drive and detector transducers. These corrections are well known for the radial resonance modes $(l=0, n=2,3,4,5$, ...). Furthermore, the radial modes are only weakly affected by small deviations from a perfect spherical geometry. Thus, the radial modes are preferred for determination of gas speed of sound $[1,2,7]$. In addition, the non-radial modes may exhibit multiple peaks further complicating their use in measuring sound speed. The corrections require geometric information for the resonator, as well as estimates for the thermodynamic and transport properties of the gas. The corrections are generally less than $0.01 \%$ in sound speed, so relative uncertainties in gas properties of $5 \%$ propagate to uncertainties of the order of $0.0005 \%$ in measured sound speed in such cases. 
At low pressures, the most significant correction for the radial modes accounts for the thermal boundary layer. The thermal boundary layer correction is a function of temperature, $T$, and pressure, $p$, given by

$$
(\Delta f-i g)_{\mathrm{Th}}=-(1+i)\left(\frac{(\gamma-1)}{2 r}\right) \sqrt{\frac{\alpha f}{\pi}}+i\left(\frac{(\gamma-1)}{2 r^{2}}\right)\left(\frac{\alpha}{\pi}\right)+\frac{(\gamma-1) f l_{\mathrm{a}}}{r},
$$

with gas properties: $\gamma$, the ratio of constant pressure heat capacity $C_{p}$ to constant volume heat capacity $C_{V} ; \alpha$, the thermal diffusivity; and $l_{\mathrm{a}}$, the thermal accommodation length. The thermal accommodation length is given by

$$
l_{\mathrm{a}}=\left(\frac{\lambda}{p}\right) \sqrt{\frac{\pi M T}{2 R}}\left(\frac{C_{V}}{R}+\frac{1}{2}\right)^{-1}\left(\frac{2-h}{h}\right),
$$

where $\lambda$ is the thermal conductivity of the gas, $M$ is its molar mass, $R$ is the molar gas constant, and $h$ is the thermal accommodation coefficient, which depends on the gas and on the resonator material and its finish. In this work, we have assumed $h=0.87$ for argon on stainless steel [9, 10], and $h=1.00$ for the other molecules based on the work of Goodman.[9]

At elevated pressures, the most significant corrections account for the radial resonance of the spherical shell of the resonator. For an isolated concentric spherical shell that is radiating to gas outside the sphere this can be directly calculated.[2] This frequency shift, $\Delta f_{\text {Sh }}$, may be approximated by

$$
\Delta f_{\mathrm{Sh}}=\frac{-f \rho w^{2} C_{i}}{\left(1-\left(\frac{f}{f_{i}}\right)^{2}\right)}
$$

where $C_{i}$ is the mechanical compliance (defined in eq 9) of the spherical shell with internal pressure, and $f_{\mathrm{i}}$ is a resonant frequency of the shell for this radial pressure dilation. Our resonator shell is more complicated with a massive flange held together with 12 bolts (see section 2.2). It is expected that the compliance normal to the plane of this flange is less than it is in-line with the flange, and multiple shell resonant frequencies have been observed. In addition, the spherical shell is enclosed in a copper sleeve rather than radiating into gas. Estimated values for the $C_{i}$ and $f_{\mathrm{i}}$ were used as starting points for this correction. Improved values were found from resonance scans for argon gas during the calibration process. It was found that this correction was very sensitive to values for $f_{\mathrm{i}}$ for radial resonance modes with frequencies near each shell resonance frequency. This allowed us to get a better value for each $f_{\mathrm{i}}$, but it was found that this correction 
increased the uncertainty of radial resonance modes near the shell resonant frequencies such that they often needed to be discarded as outliers.

Corrections were also made to account for the two filling ports, the excitation transducer, and the detection transducer $[1,2,7]$. The form of these corrections is given by

$$
\left(\Delta f_{0}+i g_{0}\right)=\left(\frac{w}{2 \pi r}\right)\left(\frac{\Delta S}{4 \pi r^{2}}\right) i y_{0},
$$

where $\Delta S$ is the surface area of the opening and $y_{0}$ is the acoustic input admittance of the opening. These corrections were generally small at all conditions due to the small size of the ports and transducers relative to the internal surface area of the sphere. Since each of these ports has a specific resonance frequency, it is possible that a specific radial mode of the sphere will occasionally overlap the resonance frequency of one of these ports. When this occurs, the sound speed from that mode appears as an outlier relative to the other, more-reliable modes, and so it was discarded.

Finally some gases, such as methane and carbon dioxide, require correction for dispersion associated with vibrational relaxation of the gas $[2,7]$. The dispersion correction is given by

$$
\Delta f_{d i s}=f_{0, n}\left[\frac{1}{2}(\gamma-1) \Delta(\omega \tau)^{2}\left(1-\frac{\Delta(1+3 \gamma)}{4}\right)\right],
$$

where $\omega=2 \pi f, \Delta$ is the ratio of vibrational heat capacity to total heat capacity (can be estimated from heat capacity of the ideal gas as in eq 13 or vibrational spectroscopy), and $\tau$ is the experimental vibrational relaxation time, typically obtained from the excess half-width of the acoustic resonance peak. $[2,7,11]$

The sphere radius is a function of both the temperature and pressure of the resonator. There are normally small variations in the sphere radius over its entire inner surface. The effective sphere radius was calibrated as a function of temperature and pressure with sound speed measurements on high-purity argon gas as described in section 3.2.

The ideal-gas heat capacity can be determined from the acoustic virial equation

$$
w^{2}=\frac{R T \gamma^{\mathrm{pg}}}{M}+A_{1}(T) \cdot p+A_{2}(T) \cdot p^{2}+\cdots,
$$

where 
Unfortunately, for a complex molecule, such as the fluids studied here, $\gamma^{\mathrm{pg}}$, is close to 1 so $\left(\gamma^{\mathrm{pg}}-1\right)$ is small and eq 8 indicates that uncertainty from extrapolation of eq 7 to zero pressure to obtain $\gamma^{\mathrm{pg}}$ leads to increased uncertainty in the ideal-gas heat capacity in such cases. An alternative approach is to analyze the sound speed data at each temperature and pressure in a multiproperty fitting process with other available data such as density, vapor pressure, heat capacity, etc. to develop an equation of state, including the ideal-gas heat capacity, for the measured fluid. Our colleagues used this second approach to determine the ideal-gas heat capacity of 1,1,1,2,2,3,3heptafluoro-3-methoxypropane (RE347mcc) [12] and of trans-1,3,3,3-tetrafluoropropene (R1234ze(E)) [13] from the sound-speed data reported here.

\subsection{Description of instrument-resonator}

A photograph of the entire instrument is shown as figure 1. It consisted of the following key components: (1) the resonator cell, which constituted the measuring system for sound speed; (2) a vacuum thermostat system that enclosed the resonator cell, incorporating fluid cooling and electrical heating; (3) pressure- and temperature-measuring instruments; (4) a computer that controlled the entire system and recorded the experimental data; and (5) auxiliary systems, including a sample charging manifold and a vacuum system.

The spherical-resonator cell consisted of thick-walled upper and lower hemispheres made from type 321 stainless steel that were joined with a twelve-bolt flange along the central plane of the sphere. Figure 2 shows the assembled resonator, and figure 3 shows the inside of the two hemispheres. The spherical resonator had a nominal internal diameter of $80 \mathrm{~mm}$ and a nominal external diameter of $120 \mathrm{~mm}$. The resonator sphere was designed as a pressure vessel for operation at temperatures from $(80$ to 500$) \mathrm{K}$ with pressures up to $40 \mathrm{MPa}$. (Operation at cryogenic temperatures would require a liquid-nitrogen flow system; this was not implemented for the present measurements, and the lower temperature limit was $265 \mathrm{~K}$ using a circulating bath.) The maximum pressure for the present measurements was $10 \mathrm{MPa}$. The sealing gaskets for the main flange, upper and lower filling port flanges, and the drive and detector transducer flanges were made from polyimide.

The sound transducer ports were located in the top hemisphere, each at a 45 degree angle from the upper filling port. This positioned them at a 90 degree angle from each other as was accepted to reduce interference between the $(0,2)$ radial mode and the $(1,3)$ mode at the time it was constructed in 1996.[2, 7] It is now known that the optimum separation angle is about $39.2^{\circ}$ to reduced interferences between these modes.[1] These transducers are shown in figure 4; they are 
based on the design of Trusler and Zarari [3]. Each transducer consisted of a solid-dielectric, capacitance-type membrane of polyimide that was metalized with gold on the side facing the resonator cavity. Each circular membrane was held near its outer diameter with a glass spacer that was tensioned with a spring, while a spring-tensioned plunger/electrode pressed against the polyimide (electrically insulated) side inside the spacer. The electrode spring made electrical contact between the plunger/electrode and a high-pressure feedthrough pin in each transducer flange. Thus, each gold metalized layer was at the cell ground relative to the potential of each plunger/transducer feedthrough pin. The plunger/electrode had six small holes drilled into its face to provide gas cushioning between the membrane and the electrode for improved sensitivity to sound waves [7].

The sinusoidal excitation voltage was provided by a synthesized function generator, with this voltage amplified by an audio transformer $(32 \times$ gain). This drive voltage generated sound in the resonator at twice the drive frequency. The membrane in the detector transducer was polarized (up to $200 \mathrm{VDC}$ ) to generate a voltage as sound waves moved the membrane relative to the electrode. The detector output was connected with a triaxial cable to a bootstrapped JFET operational amplifier (unity gain) that buffered the signal; this op-amp (LF356N or OP42EZ) was located in the vacuum space of the thermostat and held near ambient temperature. Figure 5 shows a schematic diagram of this amplifier circuit. The buffered output from the op-amp drove the intermediate coaxial conductor to the transducer voltage to nearly eliminate capacitive coupling between the inner signal conductor and the intermediate shield. The outer coaxial shield was grounded. The buffered output from the detector/JFET amplifier was then amplified and recorded as in-phase and quadrature signals with a digital lock-in amplifier.

A measurement of sound speed consisted of a series of frequency scans, with both increasing and decreasing frequency, centered about each of the three to five lowest radial modes $(0,2)$ through $[(0,4),(0,5)$ or $(0,6)]$ at each controlled temperature and pressure. Consistency was checked by comparison of the sound speed from each mode, differences between increasing versus decreasing frequency scans, and from multiple replications of this entire process.

We developed a peak-finding algorithm that largely automated the process of finding and measuring the resonances; the algorithm also adjusted the output level of the function generator and the sensitivity of the lock-in amplifier. The spectrum of resonance peaks was complex, but the relative position of each peak scaled with the sound speed of the gas, so locating one peak allowed the frequency of all the other peaks to be estimated. The frequency of a specific mode, such as the $(0,2)$, was estimated using equation 1 based on an estimate of the sound speed at the temperature and pressure of the measurement. (This estimate was calculated with a preliminary equation of state, if available, or on the basis of the previous isotherm measured on that fluid.) 
This, along with an estimate of the half-width of the resonance peak, was often all that was necessary for our peak-finding algorithm. For difficult cases, a manual scan was occasionally necessary to better specify the frequency and half-width of the starting resonance peak. The peak finding algorithm scanned 25 points, centered at the specified resonance frequency, with frequency increments that spanned the expected frequency range of the peak. The data were then analyzed to obtain better estimates of the resonance frequency and half-width of the peak. These improved estimates were used to adjust the range of the next scan and to adjust the frequency generator and lock-in amplifier, if needed. This process was repeated twice before the final resonance scan was measured and recorded.

The in-phase and quadrature amplitudes of the resonance scan were fitted as a function of frequency to a vibration model as described by Ewing and Trusler [14] and Trusler [7]. The data at increasing frequency were fitted independently from the data at decreasing frequency to check for possible hysteresis for each radial mode. The resonance frequencies from these fits were used with the sphere radius calibration and the working equation (equation 1) to obtain experimental values for the sound speed of the fluid as a function of the temperature and pressure of the fluid.

\subsection{Description of instrument-thermostat and pressure measuring system}

The thermostat provided a uniform and controlled temperature environment for the resonator and fluid sample. The thermostat was a multi-layer, vacuum-insulated type, and a schematic is shown as figure 6. The innermost element was the resonator sphere; it was controlled within $5 \mathrm{mK}$ of a constant temperature. The resonator was surrounded by a close-fitting, multi-part copper sleeve; figure 7 shows the resonator and the copper sleeve. The copper sleeve served to reduce temperature gradients in the stainless-steel resonator sphere. A total of eight small cartridge-type electric heaters were inserted into wells drilled into the sleeve. Two $25 \Omega$ standard-grade platinum resistance thermometers (SPRT) were located within the copper sleeve. The control SPRT was located in the upper half of the copper sleeve, while the cell SPRT was located in the flange of the resonator sphere. The sphere and copper sleeve were hung from stainless-steel support tubes with relatively small heat conduction.

The next level of the thermostat was the "isothermal shield." A fluid channel in the top copper disk of the shield allowed operation at temperatures below ambient by flowing fluid from a temperature-controlled circulating bath. The shield side was fabricated from a length of copper pipe, which was attached to the top disk with screws. Electric heaters were installed in both the top disk and side. An intermediate "tempering ring" served to reduce spurious heat conduction between the resonator cell and the isothermal shield along heater wires, SPRT leads, capillary gas filling tubes, and the support tubes. The tempering ring was copper and had its own heater 
and could be controlled based on the temperature difference between the resonator cell and intermediate ring as measured with differential thermocouples. The support tubes between the isothermal shield and tempering ring are offset from those supporting the resonator cell by 60 degrees; this reduced the temperature differences between each of the three support tubes that suspended the tempering ring and, thus, reduced temperature gradients in the resonator cell. Figure 8 shows the top disk of the isothermal shield, the intermediate tempering ring, and the resonator cell with isothermal copper sleeve; the main part of the isothermal shield has been removed in figure 8 .

The shield was surrounded by passive radiation shields of aluminum foil. The outermost element of the thermostat was the vacuum chamber. The vacuum system consisted of an oil diffusion pump and a liquid-nitrogen cold trap backed by a mechanical vacuum pump. Feedthroughs for the electrical and fluid connections were located in the top plate of the vacuum chamber.

The temperature of the resonator sphere was measured by a $25 \Omega$ standard platinum resistance thermometer (SPRT) in a thermowell drilled through the outer flange of the sphere. This "cell SPRT," which determined the temperature reported in the results, was measured with an AC resistance bridge. The $25 \Omega$ reference resistor for the bridge was thermostatted at $37.0 \pm 0.1{ }^{\circ} \mathrm{C}$ in a small enclosure.

The electric heaters on the sphere and shields were controlled with the modified proportionalintegral-derivative (PID) control algorithm of Hust et al. [15]. The cell heaters were controlled using the output of a $25 \Omega$ SPRT; this "control SPRT" was installed in a thermowell drilled into the copper sleeve. The heaters on the shield were controlled using the output of type T (copperconstantan) thermopiles, with their reference junctions in thermowells adjacent to the control SPRT. These transducers were measured with a pair of voltmeters. The shield was controlled at a temperature approximately $1 \mathrm{~K}$ below the sphere temperature. The sphere temperature was stable to $5 \mathrm{mK}$ or better, and the shield temperature was stable to $10 \mathrm{mK}$ or better.

The pressure of the sample was measured with one of three vibrating-quartz-crystal-type pressure transducers with full-scale ranges of $0.7 \mathrm{MPa}, 6.2 \mathrm{MPa}$, and $41 \mathrm{MPa}$. The transducers were in direct contact with the sample - no differential pressure diaphragm was used. They were housed in a close-fitting aluminum block, which was thermostatted at $40.0 \pm 0.1{ }^{\circ} \mathrm{C}$.

For high-pressure samples (argon, methane, ethane, R1234ze(E)) the fluid sample was loaded into the resonator directly from the sample bottle. For RE347mcc, which had a relatively low vapor pressure, the fluid sample was loaded into the resonator sphere using a hand-operated, high-pressure, piston-type pump. The sample cylinder was heated to increase the vapor pressure, and the piston was slowly withdrawn to fill the pump. The desired quantity of liquid sample was 
then pushed into the system, where it vaporized upon entering the evacuated resonator sphere. The pressure manifold for sample handling and pressure measurement is shown in figure 9 .

After the sample was loaded, a test run was started; temperatures and pressures were scanned every $60 \mathrm{~s}$. Once the set-point conditions were reached, an additional 60 min was allowed for complete equilibration before starting the peak-finding algorithm. The radial modes were then scanned and recorded. Typically, three replicates were measured at a given $(T, p)$ state point, and then a portion of the sample was vented into a waste bottle to reduce the pressure to the next state point.

\section{Results}

Measurements of the speed of sound of five fluids were made during this work. The sound speed of argon gas is well known, and it was used as a calibration fluid to characterize the radius of the spherical resonator at temperatures from (265 to 500) $\mathrm{K}$ with pressures to $1 \mathrm{MPa}$. Measurements at pressures up to $10 \mathrm{MPa}$ served to check the pressure dilation of the sphere. The sound speeds of methane and ethane are also well known, and they were used as verification fluids at temperatures from ( 280 to 485$) \mathrm{K}$. Finally, the resonator apparatus was used to measure the sound speed of 1,1,1,2,2,3,3-heptafluoro-3-methoxypropane (RE347mcc) at temperatures from (325 to 500) $\mathrm{K}$ with pressures up to 1.6 MPa; trans-1,3,3,3-tetrafluoropropene (R1234ze(E)) was measured at temperatures from ( 280 to 420$) \mathrm{K}$ with pressures up to $2.8 \mathrm{MPa}$. These sound speed data have been used along with density and other thermodynamic data to develop accurate equations of state for these fluids, as reported elsewhere [12, 13].

\subsection{Characterization of gas samples}

The purity of the gas samples used in this work is summarized in table 1. The argon used in the sphere calibrations was "ULSI-grade" (Matheson Tri-Gas, Inc.) with a certified molar purity of 0.999999 . A cylinder analysis by the supplier indicated impurities of nitrogen at $0.49 \mathrm{ppm}$, oxygen at $0.11 \mathrm{ppm}$, and water at $0.17 \mathrm{ppm}$. Carbon dioxide, carbon monoxide, and methane were not detected at the level of $0.1 \mathrm{ppm}$. Our own analysis by gas chromatography was consistent with the supplier's analysis.

The methane used in the performance verification tests had a molar purity of 0.99999 by the supplier's analysis (Matheson Tri-Gas, Inc.). The ethane was 0.9999 pure (molar basis) by the supplier's specification (Matheson Tri-Gas, Inc.). These purities were verified by our own analysis by gas chromatography/mass spectrometry.

The RE347mcc used in the testing was 0.999 pure (basis not specified) by the supplier's analysis (3M Electronics Markets Materials Division). Our own analysis by gas chromatography/mass 

molar purity of 0.9989 and showed two impurities: 0.00047 mole fraction of a fluorinated hydrocarbon (not otherwise identified) and a second 0.00067 mole fraction impurity, possibly a chlorinated ethene. The water content, determined by a Karl Fischer titration, was 0.000013 mole fraction. For all measurements, the sample was used as received, except that it was degassed with multiple cycles of freezing the sample, evacuating the vapor space, and thawing. The pressure over the frozen material on the final freeze-pump-thaw cycle was $1 \cdot 10^{-4} \mathrm{~Pa}$.

The supplier's analysis of the sample of R1234ze(E) indicated a molar purity of 0.99993. Our own analysis by gas chromatography combined with mass spectrometry and infrared spectrophotometry revealed only very small impurity peaks that were too small to permit identification. This sample was also degassed to a final pressure of $1 \cdot 10^{-4} \mathrm{~Pa}$.

\subsection{Calibration of resonator diameter with argon}

The diameter of the spherical resonator at zero pressure was calibrated as a function of temperature from measurements of the sound speed of argon gas, which is known with very low uncertainty (see section 4.3). Measurements of sound speed of argon were made along isotherms at pressures less than $1 \mathrm{MPa}$ for the calibration. Measurements at pressures up to $10 \mathrm{MPa}$ were used to verify the calculated pressure dilation. The sound speed data were corrected for pressure dilation of the sphere given its geometry and the mechanical properties of type 321 stainless steel. The expansion of the sphere diameter, $d$, with internal pressure relative to its value at zero pressure, $d_{0}$, can be calculated from the shell compliance $C_{0}$

$$
\frac{d}{d_{0}}=1+C_{0} p
$$

The shell compliance is obtained from

$$
C_{0}=\frac{\left[(1+\sigma) q^{3}+2(1-2 \sigma)\right]}{\left.\left[2 E\left(q^{3}-1\right)\right)\right]}
$$

where $q$ is the ratio of the outside diameter to the inside diameter of the sphere, $\sigma$ is Poisson's ratio and $E$ is Young's modulus of the type 321 alloy stainless steel. With $q=1.5$ for the present sphere, the sphere diameter increases linearly by about $60 \mathrm{ppm}$ with an internal pressure of $10 \mathrm{MPa}$. The experimental measurements of the sound speed of argon at pressures to $10 \mathrm{MPa}$ were consistent with these pressure dilation calculations (see Supplemental Data for a comparison of argon data relative to the calibration at pressures up to $10 \mathrm{MPa}$ ). 
The sphere radius at zero pressure $d_{0}$ was determined by fitting the calibration data to the expression

$$
d_{0}(T)=\frac{w_{\mathrm{EOS}}(T, p) v_{0, n}}{\pi f_{0, n}(1+C p)},
$$

where $w_{\mathrm{EOS}}$ is the speed of sound given by the argon equation of state, $v_{0, n}$ is the eigenvalue for the $(0, n)$ radial mode, $f_{0, n}$ is the measured frequency (corrected for boundary layer, fill port, transducer, and shell compliance effects, as described in section 2.1), and $p$ is pressure in Pa.

Since the range of the argon calibration data was limited to a maximum of $1 \mathrm{MPa}$, the magnitude of the correction for pressure dilation was less than $6 \mathrm{ppm}$ and uncertainty in this correction is insignificant during the calibration process. (The uncertainty in the argon equation of state increases with pressure, however, and this effect must be included in the uncertainty analysis, as discussed in section 4.) The sound speed extrapolated to zero pressure of three or more isotherms was fitted to a quadratic function

$$
d_{0}=d_{0}^{T_{0}}\left(1+d_{1}\left(T-T_{0}\right)+d_{2}\left(T-T_{0}\right)^{2}\right)
$$

Equation 12 gives the sphere diameter at zero pressure, relative to its diameter at zero pressure and the reference temperature of $T_{0}=300 \mathrm{~K}$.

The sphere diameter was calibrated with four series of measurements of the sound speed of argon. These test series were conducted: 1) November to December 2011, 2) March 2012, 3) August to September 2012, and 4) April 2014 over a combined temperature range of (265 to 500) K. Figure 10 summarizes the results of these calibrations. (The baseline in the figure is the data of April 2014, series four.) Series one, two, and four are seen to agree very well; the third series (August to September 2012) measurements are in good agreement near $T_{0}=300 \mathrm{~K}$, but show systematic deviations at lower and higher temperatures. These measurements were carried out following removal of the SPRTs for calibration, and we suspect that, upon reinstallation, the SPRT readings were affected by thermal contact of their leads with other lead wires that were near ambient temperature. (The SPRTs were located in close fitting holes in the cell but the heat transfer between the cell and the SPRTs was reduced in the high vacuum conditions during measurements, and so, the reading could have been affected by heat conduction down the leads.) We installed the tempering ring (for the SPRT leads) and the thermal anchor in the top isothermal shield (for the acoustic transducer leads) after observing this effect. The fourth series (April 2014) measurements were carried out after installation of the tempering ring, and they are seen to agree well with the first two calibration series. 
The measurements on RE347mcc were conducted shortly after the second calibration series, and the measurements on R1234ze(E) were conducted after the third series. The two sets of validation measurements on methane and ethane were conducted before and after the April 2014 calibrations. The calibration results suggest that the only the measurements between August 2012 and April 2014 (i.e., those on R1234ze(E) and the March 2013 measurements on methane and ethane) were affected by conduction down the leads. But even for these measurements, the sound speeds are relative to argon and any systematic error in the temperature measurement would be canceled, to first order, by the calibration; the results for methane and ethane (discussed in section 3.3) confirm this.

The argon calibrations spanned 29 months, and this time period included cycling the resonator numerous times over it's full operating temperature range, with pressures up to $10 \mathrm{MPa}$. The constancy of the diameter calibration at $T_{0}=300 \mathrm{~K}$ demonstrates the mechanical stability of the sphere.

\subsection{Verification measurements with methane and ethane}

The performance of the entire system can be demonstrated by comparing measurements on wellcharacterized gases with high-accuracy literature data and equations of state. This was done using methane and ethane. The dispersion correction must be applied to the measured resonance frequencies for methane, but it is much less significant for ethane. The fractional vibrational heat capacity needed in equation 6 is obtained from the molar ideal gas heat capacity with

$$
\Delta=\frac{C_{p}^{0}-4 R}{C_{p}^{0}},
$$

where $C_{\mathrm{p}}{ }^{0}$ is the ideal gas heat capacity and $R$ is the molar gas constant. We calculated $\Delta$ and $\gamma$ from the equations of state of Setzmann and Wagner[18] for methane and Bücker and Wagner[19] for ethane; values for the relaxation time $\tau$ were available from definitive sound speed measurements of methane[3] and ethane [20].

For each gas, measurements of the $(0,2)$ through $(0,4)$ modes were carried out at a pressure of approximately $500 \mathrm{kPa}$ and a temperature range of (280 to 480 ) $\mathrm{K}$ for methane and (280 to 485) K for ethane. Two separate sets of tests were carried out in March 2013 and July 2014 (before and after installation of the tempering ring). Our results are presented in figure 11, where the baselines are the equations of state of Setzmann and Wagner [18] for methane and Bücker and Wagner [19] for ethane. Also shown in figure 11 are the high-accuracy experimental data of Trusler and Zarari [3] and Ewing and Goodwin [21] for methane and Estrada-Alexanders and Trusler [20] for ethane; here we have plotted the literature data only at $p \leq 2 \mathrm{MPa}$. 
Our methane data are in very good agreement with the equation of state and literature data at $T \leq 320 \mathrm{~K}$, but show increasing systematic deviations at higher temperatures, although they are within our combined expanded uncertainty (as discussed in section 4). There are only small differences between the 2013 and 2014 measurements. The RMS deviation of both our 2013 and 2014 data sets for methane from the EOS is $0.0112 \%$, compared to an RMS deviation of $0.0016 \%$ for the data of Trusler and $0.0021 \%$ for the data of Ewing and Goodwin at $p \leq 2 \mathrm{MPa}$. The data of Ewing and Goodwin were used in developing the EOS, but the data of Trusler and Zarari were not. Setzmann and Wagner [18] estimate the expanded uncertainty in the methane equation of state for vapor-phase speeds of sound as $0.03 \%$.

Our 2014 ethane measurements are in excellent agreement with the data of Estrada-Alexanders and Trusler [20] over the full temperature range of overlap, while the 2013 measurements show slightly larger deviations. The RMS deviations of our 2013 and 2014 ethane data from the EOS are $0.0070 \%$ and $0.0054 \%$, respectively, compared to an RMS deviation of $0.0044 \%$ for the data of Estrada-Alexanders and Trusler at $p \leq 2 \mathrm{MPa}$. The data of Estrada-Alexanders and Trusler were used in developing the EOS. Bücker and Wagner [19] estimate the expanded uncertainty in the ethane equation of state for vapor-phase speeds of sound as $0.02 \%$.

\subsection{Sound speed of RE347mcc}

The measured data for the sound speed of RE347mcc are shown in figure 12 along with the sound speed calculated with the equation of state (EOS) of Zhou and Lemmon [12] The measurements were carried out along isotherms from (325 to 500) K. For each isotherm, the measurements extended from a pressure equal to approximately $80 \%$ of the vapor pressure at that temperature down to a pressure of (0.06 to 0.07) $\mathrm{MPa}$, with four to nine pressures measured for each isotherm. For temperatures above the critical temperature of $437.7 \mathrm{~K}$, the maximum pressure was $1.6 \mathrm{MPa}$. Three or more replicates, comprising measurements of the $(0,2)$ through $(0,4)$ radial modes, were made at each $(T, p)$ state point.

The measured sound speed was found to increase with sample residence time for RE347mcc. The increase in sound speed became more apparent at temperatures above $400 \mathrm{~K}$ and at pressures below $500 \mathrm{kPa}$, see figure 13 . This was likely due to sample decomposition since measurements on argon gas before and after the measurements on RE347mcc did not indicate any significant shift in the resonator diameter or mechanical properties. Five isotherms at (400, 425, 450, 475, and 500) $\mathrm{K}$ were analyzed for the rate of change of measured sound speed of RE347mcc. At a fixed temperature, the relative rate of change of sound speed with elapsed time $(\partial w / \partial t)_{T}$ was inversely proportional to pressure: 

process within the sphere. Figure 14 shows the pressure dependence for a representative isotherm at $500 \mathrm{~K}$.

It was found that the coefficient $a$ increased exponentially with respect to temperature based on an examination of the isotherms from $400 \mathrm{~K}$ to $500 \mathrm{~K}$ :

$$
a=b \exp (c T)
$$

An exponential increase with respect to temperature is characteristic of chemical reactions with first-order kinetics. Thus, the measurements suggest that a surface-catalyzed reaction may have occurred during the speed-of-sound measurements on RE347mcc. The same behavior would be expected for the diffusion of a gas out of the sealing gaskets; but, the gas previously measured in the system was argon, which would not be expected to diffuse into the polyimide gaskets, and the entire system was thoroughly evacuated between samples.

The fit of $a(T)$ at these five temperatures to an exponential function in $T$ is shown in figure 15. This figure shows that the rate of change should rapidly decrease with temperature for temperatures below $400 \mathrm{~K}$. The values for $a(T)$ given by this fit are more reliable than it is possible to obtain from an analysis of the data for these lower temperature isotherms. The corresponding coefficients for RE347mcc are $b=8.85 \times 10^{-9} \mathrm{kPa}^{-1} \mathrm{hr}^{-1}$ and $c=0.0271 \mathrm{~K}^{-1}$.

Regardless of the mechanism of the sound speed shift, it is possible to correct for the shift by fitting (for each series of measurements along an isotherm) the measured shift in sound speed to the two-parameter function of pressure and temperature given above. The shift in sound speed for any incremental measurement after the initial filling of the resonator for a given isotherm depended on the temperature-pressure history of the sample and is given by

$$
w=w_{\text {meas }}\left(t_{N}\right)-\sum_{i=1}^{N}\left[\frac{\partial w\left(T_{i}, p_{i}\right)}{\partial t}\right]\left(t_{i}-t_{i-1}\right)=w_{\text {meas }}\left(t_{N}\right)-\sum_{i=1}^{N}\left[\frac{b \exp \left(c T_{i}\right)}{p_{i}}\right]\left(t_{i}-t_{i-1}\right) \text {. }
$$

where $t_{0}$ is the time when the (fresh) sample was loaded into the resonator. The overall correction to sound speed was generally small for the higher-pressure measurements where the rate of change was small and less time had elapsed since filling the resonator. As the pressure was decreased along the isotherm, the correction to the measured sound speed increased due to longer residence time and increasing rate of change in the sound speed. 
The corrected sound speed data for RE347mcc are given in table A.1 of the appendix along with their combined, expanded uncertainty at each measured temperature and pressure. Table A.1 also gives the deviations from the measured values with those calculated from the equation of state of Zhou and Lemmon [12] These deviations are also shown in figure 16. Details of the stability correction are given in the Supplementary Data.

\subsection{Sound speed of R1234ze(E)}

The measured data for the sound speed of R1234ze(E) are shown in figure 17 along with the sound speed calculated with the equation of state (EOS) of Thol and Lemmon [13]. The measurements were carried out along isotherms from (280 to 420) K. For each isotherm, the measurements extended from a pressure equal to approximately $80 \%$ of the vapor pressure at that temperature down to a pressure of $(0.08$ to 0.20$) \mathrm{MPa}$, with three to eleven pressures measured for each isotherm. For temperatures above the critical temperature of $382.5 \mathrm{~K}$, the maximum pressure was up to $2.8 \mathrm{MPa}$. Three or more replicates, comprising measurements of the first three radial modes, were made at each $(T, p)$ state point.

The measured sound speed was found to increase with sample residence time for R1234ze(E) similar to that described in section 3.4 for RE347mcc. However, for R1234ze(E) this increase was about $10 \%$ of that observed for RE347mcc. The measured data were corrected using the technique described for RE347mcc and the corresponding coefficients needed in equation 13 for $\mathrm{R} 1234 \mathrm{ze}(\mathrm{E})$ are $b=5.63 \times 10^{-10} \mathrm{kPa} \cdot \mathrm{hr}^{-1}$ and $c=0.0337 \mathrm{~K}^{-1}$.

The corrected sound speed data for R1234ze(E) are given in Table A.2 of the appendix along with their combined expanded uncertainty at each measured temperature and pressure. Table A.2 also gives the deviations between the measured values and those calculated value from the equation of state of Thol and Lemmon [13] at each measured state point. Details of the stability correction are given in the Supplementary Data. Only one data set by Kano et al. [22] is available in the literature for the vapor sound speed of R1234ze(E) at temperatures from $278 \mathrm{~K}$ to $353 \mathrm{~K}$. These deviations are shown in figure 18, demonstrating agreement between the present measurements and those of Kano et al. [22] within their expanded uncertainties.

\section{Uncertainty of the Measurements}

The measurement of the speed of sound essentially reduces to the measurement of geometry (the sphere diameter) and time (i.e., the period of the resonant sound wave). Both geometry and time can be measured with extremely low uncertainties, and the potential for low uncertainties is one of the major advantages of measuring the speed of sound over other quantities (such as heat capacity) that would yield similar thermodynamic information for the purposes of fitting an 

considered carefully. These can be grouped into uncertainties resulting from the resonance signal itself (i.e., timing uncertainties, noise and fitting of the signal); corrections to the measured resonance frequencies; determination of the geometry (i.e., the calibration and stability of the sphere diameter); and the determination of the thermodynamic $(T, p, x)$ state-point. In this discussion, we consider standard $(k=1)$ uncertainties and apply a coverage factor when combining the individual uncertainty components.

\subsection{Uncertainty of the resonance signal}

The resonance signal was detected with a digital lock-in amplifier, which was synchronized with the time base of the function generator. The function generator had a temperature-controlled quartz-crystal oscillator with a relative "stability" uncertainty less than $0.01 \times 10^{-6}$ with long-term drift less than $0.001 \times 10^{-6}$ per day, according to the manufacture's specification. Assuming the resonator is calibrated with argon yearly, the relative uncertainty in the time base would effectively be less than $0.365 \times 10^{-6}$. The uncertainty in the speed of sound is directly proportional to this uncertainty, and so, the uncertainty from timing was negligible.

The signal to the lock-in amplifier was typically in the range of (50 to 1000$) \mu \mathrm{V}$ and the $\mathrm{A} / \mathrm{D}$ converter had a resolution of 16 bits or 1 part in 65536 of the full-scale range. The stability of the resonance is very sensitive to small changes in temperature and pressure, especially near sharp resonance peaks (which is to say, precisely where we are measuring). The signal was also sensitive to outside vibrations; in other words, the "noise" in the electrical signal may be due, in part, to acoustic noise in the laboratory. The resonance in the sphere took a finite time to build to a steady-state value, and if insufficient settling time was allowed between frequency steps, the apparent resonance peak would be skewed; this would be revealed by a difference in the fitted peak frequency between the ascending and descending frequency scans for a given mode. The uncertainties from these effects are included in the weighted average discussed in section 4.5.

\subsection{Corrections to the measured frequencies}

The resonant frequencies were corrected for several effects, as discussed in section 2.1. The uncertainty due to the boundary layer correction is proportional to the uncertainty in the thermophysical properties required in equations 2 and 3. This correction was relatively large for argon (up to $0.015 \%$ ), but the properties for argon are well known (uncertainty of $1 \%$ or lower). The corrections for R1234ze(E) and RE347mcc were smaller (maximum of $0.0025 \%$ for $\mathrm{R} 1234 \mathrm{ze}(\mathrm{E})$ ), but the thermophysical properties have larger uncertainties (up to $10 \%$ for thermal conductivity). The uncertainty for the boundary layer correction was less than $0.0002 \%$ for RE347mcc and R1234ze(E). 
The geometry of our flanged design is considerably more complex than the simple, concentric spheres assumed in equation 4, and thus the shell resonance correction is unreliable at frequencies near the resonant frequency of the sphere $f_{0}$. We estimated the uncertainty as $10 \%$ of the correction at frequencies far away from $f_{0}$, and we discarded radial modes when this correction would have exceeded $0.01 \%$ (a magnitude that was not normally encountered in the $(0,2)$ to $(0,4)$ radial modes). Thus, the uncertainty from the shell resonance correction was $0.001 \%$ or less. The corrections due to the transducer and filling ports were always less than $0.0001 \%$, and thus the uncertainties in these corrections were negligible.

Dispersion effects can affect the resonance, depending on the molecule being measured [2, 7 , 11]. These effects are not present for a monoatomic fluid (e.g., argon), and are negligible for complex molecules, such as R1234ze(E) and RE347mcc. They can be significant for simple molecules, such as methane and ethane, especially at low densities. The largest effect observed in the present work was a correction of $0.33 \mathrm{~Hz}$ for the $(0,4)$ mode in methane at $T=320 \mathrm{~K}$ and $p=472 \mathrm{kPa}$, which was a relative effect of $17 \times 10^{-6}$. Thus, the uncertainty from dispersion effects was quite small.

\subsection{Diameter of spherical cavity}

The diameter of the spherical cavity was determined by calibration measurements with argon. The uncertainty in the argon speed of sound in the limit of zero pressure is essentially that of the molar gas constant, or $0.91 \times 10^{-6}$ [23]. But we used measurements at pressures up to $1 \mathrm{MPa}$ in our calibrations, and thus the uncertainty in the argon equation of state must also be considered. Over our temperature range of (265 to 500) $\mathrm{K}$ and pressures up to $1 \mathrm{MPa}$, Tegeler et al. [24] state the uncertainty of their EOS as $0.02 \%$. This EOS, however, reproduces several high-quality data sets with much lower deviations. These include the data of Estrada-Alexanders and Trusler [25] with deviations of less than $0.002 \%$ over temperatures from (120 to 450) K (except near the critical temperature) and densities up to one-half the critical density (corresponding to pressures up to $19 \mathrm{MPa}$ ). The EOS represents the data of Ewing and Goodwin [26] to less than $0.003 \%$ over the temperature range of ( 255 to 300$) \mathrm{K}$, with pressures up to $7 \mathrm{MPa}$; and the data of Boyes [27] to less than $0.012 \%$ over the temperature range of (250 to 350$) \mathrm{K}$, with pressures to $10 \mathrm{MPa}$. The uncertainty of the equation of state contribution must include the experimental uncertainty of these sources, as well as how well the EOS represents the data. Thus, we estimate the standard uncertainty in the calibration arising from the EOS as $0.003 \%$. The uncertainties related to fitting the peaks and corrections to the resonance peaks (as discussed in sections 4.1 and 4.2) are applicable. The argon was of very high purity (0.999999 mole fraction), so that impurities in the gas sample had a negligible contribution to the speed of sound uncertainty. Impurities could have also come from the system itself (e.g., a previous sample), but we 
thoroughly evacuated and purged the system prior to the argon calibrations and consider this source to be negligible. The total standard uncertainty arising from the sphere calibration was estimated to be $0.0040 \mathrm{~mm}$ or $0.0050 \%$ at the reference temperature of $300 \mathrm{~K}$; it increased at temperatures away from $300 \mathrm{~K}$ (at the rate of $0.00004 \mathrm{~mm} \times(T / \mathrm{K}-300)$ ) and at higher pressures (at the rate of $0.00016 \mathrm{~mm} \times p / \mathrm{MPa}$ ).

Related to the uncertainty in the calibration of the sphere diameter is the mechanical stability of the sphere itself, i.e., did the diameter, compliance, or other properties change over time? Zarari [28], for example, discuss the annealing of their sphere after operation at high temperatures. Four sets of argon calibration data measured over the course of 29 months (a time period that included several cycles over the complete operating temperature range) had a standard deviation in $d_{0}{ }^{300 \mathrm{~K}}$ of $0.0013 \mathrm{~mm}$, and we take this as the standard uncertainty related to the stability of the sphere.

We observed repeatable and systematic differences in the speeds of sound determined from the different resonant modes. These may be due to imperfect corrections applied to account for the distorting effects of adjacent, non-radial resonant peaks in the fluid. They may also arise from deviations from a perfect spherical geometry. Zarari [28], for example, observed a total range of $0.0065 \%$ for the $(0,2)$ through $(0,6)$ modes in argon. She attributed this variation, which was repeatable over a wide range of temperature and pressure to the level of a few parts in $10^{6}$, to an imperfect sphere. The uncertainty arising from this effect is accounted for in the weighted average, as discussed in section 4.5.

\subsection{State-point uncertainty}

The uncertainty in the temperature measurement system (SPRT and its calibration, resistance bridge, and standard resistor) was estimated to be $5 \mathrm{mK}$. The SPRTs were calibrated with fixedpoint cells on ITS-90. The uncertainty in the temperature of the fluid sample also included the effect of temperature gradients, and we estimated the total temperature uncertainty to be $20 \mathrm{mK}$. The uncertainty in the pressure measurement arose from the calibration of the transducers, the repeatability and temporal drift of the transducers, and the uncertainty in the hydrostatic head correction. We estimated the standard uncertainty in the pressure measurement to be $\left(20 \times 10^{-6} \cdot p\right.$ $+0.020 \mathrm{kPa})$ for the low-range transducer, $\left(20 \times 10^{-6} \cdot p+0.15 \mathrm{kPa}\right)$ for the mid-range transducer, and $\left(26 \times 10^{-6} \cdot p+1.00 \mathrm{kPa}\right)$ for the high-range transducer. These were based on the uncertainties of the piston-gage pressure standards used for the calibration and the observed stability of the transducers when connected to vacuum. We estimate the uncertainty in the hydrostatic head correction to be $10 \%$ of the correction. The standard deviations in the observed temperature and pressure readings taken before, during, and after a resonance scan were added to these 
uncertainty estimates. The effect of these state-point uncertainties on the speed of sound is given by:

$$
u_{T, p}=\left[\left(\frac{\partial w}{\partial T}\right)^{2} u^{2}(T)+\left(\frac{\partial w}{\partial p}\right)^{2} u^{2}(p)\right]^{1 / 2}
$$

where the derivatives are estimated from the equation of state for the fluid being measured. The average state-point uncertainty for the present measurements increased with pressure and ranged from $0.002 \%$ to $0.020 \%$ for RE347mcc and from $0.002 \%$ to $0.013 \%$ for R1234ze(E). But since the argon calibrations of the sphere diameter were subject to similar systematic uncertainties in temperature and pressure, equation 17 represents a conservative estimate of the state-point uncertainty.

The state-point also depended on the composition of the sample. This included not only impurities in the fluid sample, but also contamination that might be introduced by the system and possible decomposition of the sample during the course of the testing. The effect of the composition uncertainty varied greatly among the fluids tested in this work. The argon used for the sphere calibrations reported here is a stable material and our sample was of very high purity (0.999999 mole fraction), and thus, the composition effect for argon was thought to be negligible. The purity of the methane was 0.99999 mole fraction; the likely impurities (nitrogen, ethane, and water) all have lower speeds of sound, and we estimated the uncertainty due to composition as $0.0003 \%$. The purity of the ethane sample was 0.9999 mole fraction; for this fluid the likely impurities had both higher and lower speeds of sound, and we estimated the uncertainty as $0.002 \%$. The purity of the R1234ze(E) and RE347mcc samples were 0.99993 mole fraction and 0.9989 mole fraction, respectively. The impurities were similar materials that would be expected to have similar speeds of sound. The impurities could not be identified, and the magnitude of this effect cannot be quantified; based on engineering judgment we estimate this effect as $0.01 \%$ for these two fluids. The RE347mcc and R1234ze(E), however, decomposed over the course of the testing; an adjustment to the speed of sound to $t=0$ was applied, as discussed in sections 3.4 and 3.5. We estimate the uncertainty in this adjustment to be $5 \%$ of the correction, or a maximum of $0.013 \%$ for the measurement on RE347mcc at $T=$ $500 \mathrm{~K}$ and $p=62 \mathrm{kPa}$.

\subsection{Combined uncertainty and weighted average}

The combined uncertainty $u_{\mathrm{c}}$ for a given frequency scan $i$ is given by 
where the summation is over the individual uncertainty components discussed in sections 4.1 through 4.4 and the ascending and descending frequency scans for a given radial mode are considered separately. A weighted average value of sound speed for a given $(T, p)$ state point is given by

$$
\bar{w}=\sum_{i}\left[\frac{w_{i}}{\left(u_{\mathrm{c}, i}\right)^{2}}\right] / \sum_{j}\left[\frac{1}{\left(u_{\mathrm{c}, i}\right)^{2}}\right] .
$$

This is the standard method of taking the inverse of the square of the uncertainty as the weighting factor.

The combined expanded uncertainty of $\bar{w}$ is given by

$$
U_{\mathrm{c}}=2 \cdot\left[\left(\frac{1}{N}\right) \sum_{i=1}^{N}\left(u_{\mathrm{c}, i}\right)^{2}+\left(\frac{1}{N-1}\right) \sum_{i=1}^{N}\left(w_{i}-\bar{w}\right)^{2}\right]^{1 / 2} .
$$

where the 2 is a coverage factor corresponding to an approximate $95 \%$ confidence interval and the first summation corresponds to the average combined uncertainty for the $N$ measured "modes" (where, again, the ascending and descending frequency scans for a given radial mode are considered separately). The second summation is the variance (standard deviation squared) of the sound speeds of the individual "modes;" this "between-modes" term accounts for the effects of peak fitting (half-width); insufficient settling time between frequencies; influence of adjacent, non-radial modes; and deviations from a perfect spherical geometry. The results from equation 20 are reported in tables A.1 and A.2 of the appendix.

Equations 18 through 20 generally follow the method of Paule and Mandel [29]. But, in contrast to their method, an average variance is used in the first summation of equation 20 rather than a pooled standard deviation. Our uncertainties are dominated by systematic effects, and a pooled standard deviation would be appropriate only if the errors were largely random. (Otherwise, making more replicate measurements would reduce the combined uncertainty as $(1 / N)^{0.5}$. For example, a major uncertainty is the diameter of the sphere; the actual diameter of the sphere is quite stable, but our knowledge of it is uncertain. Thus, making repeated measurements to increase $N$ would not improve our knowledge of the diameter.) 


\subsection{Summary of uncertainties}

A tabulation of the individual uncertainty components for each measured point are given in the Supplementary Data, and a summary of the uncertainties for each of the fluids is given in Table 2. The relative combined expanded uncertainty $(k=2)$ in the speed of sound ranged from $0.012 \%$ to $0.054 \%$ for the different fluids. Because of the detailed nature of the uncertainty analysis, it is not possible to simply state a value of uncertainty that would apply to all fluids and all conditions.

Table 2 shows that knowledge of the state-point can be a major source of uncertainty. Sample purity was significant for ethane, RE347mcc, and R1234ze(E) - samples with molar purities of approximately 0.9999 . Only for the methane, with a molar purity of 0.99999 , was this source relatively small. Sample instability was quite significant for the highest temperatures measured for RE347mcc. These results emphasize the importance of working with a high-purity sample and being aware of possible sample degradation during the course of the measurements. The temperature effect was moderate for all of the fluids. We expect that the temperature uncertainty will be reduced in the future as a result of reduced temperature gradients due to the "intermediate ring" (which was installed after the majority of the measurements reported here) and as we finetune the temperature control algorithm. The pressure effect varied from fluid to fluid; it was most significant for RE347mcc and R1234ze(E), which were measured much closer to the critical point (where the sound speed changes rapidly with pressure and temperature) compared to methane and ethane, which were measured well away from their critical points.

The uncertainty terms related to the sphere diameter together make up a major portion of the overall uncertainty. These will likely be lower in the future as we accumulate additional argon calibration data.

By comparison, the boundary layer and shell resonance corrections were small for all of the fluids. The dispersion correction was essentially zero, except for methane. The uncertainty due to the half-width (i.e., the uncertainty resulting from the fitting of the resonance peak) was most significant for methane. Methane also showed much larger variance for the sound speed between the different radial modes. This fluid had the highest sound speed of those measured here, and the frequency of the $(0,4)$ mode was in the range of $(20$ to 24$) \mathrm{kHz}$; this was near the resonant frequency of the shell, and so the simple model for the shell resonance (equation 4) may have been inadequate. By contrast, the difference in sound speed determined from the different modes for RE347mcc and RE1234ze(E) was as low as $0.0005 \%$; for these fluids, the frequency of the $(0,4)$ mode was in the range of $(4.5$ to 7.8$) \mathrm{kHz}$. 


\section{Discussion and Conclusions}

A spherical resonator apparatus has been developed at NIST for measurement of the sound speed of pure gases and gas mixtures. The diameter of the resonator was calibrated with measurements of the radial resonance modes of high purity argon gas. The resonator was enclosed in a highvacuum thermostat and was demonstrated over the temperature range from (265 to 500) K with pressures up to $10 \mathrm{MPa}$. The performance of the spherical resonator apparatus was validated with measurements of methane and ethane; the RMS deviations from high-accuracy equations of state were $0.0112 \%$ for methane and $0.0054 \%$ to $0.0070 \%$ for ethane. The uncertainties achieved with this system are larger than for some specialized systems, but it was intended as a generalpurpose instrument for the measurement of industrially important gases.

The spherical resonator apparatus was used to determine the sound speed of RE347mcc, which is of interest for organic Rankine power cycles and of $\mathrm{R} 1234 \mathrm{ze}(\mathrm{E})$, which is of interest for refrigeration cycles. The RE347mcc and R1234ze(E) had molar purities of $0.9989 \%$ and $0.99993 \%$, respectively, and the composition uncertainty had a small but significant impact on the overall uncertainty of the reported sound speed measurements. The average uncertainties of the measured sound speed for RE347mcc and R1234ze(E) were $0.029 \%$ and $0.041 \%$ respectively. The measured sound speeds were used, along with low-uncertainty $(p, \rho, T)$ data and other reliable thermodynamic property data, to develop thermodynamically consistent equations of state for these fluids. The RMS deviation between the present sound speed data and the equation of state of Zhou and Lemmon[12] for RE347mcc was $0.018 \%$; the deviations ranged from $-0.04 \%$ to $0.08 \%$. The RMS deviation for the R1234ze(E) data from the equation of state of Thol and Lemmon [13] was $0.041 \%$; the deviations ranged range from $0.03 \%$ to 0.05 $\%$.

\section{Associated Content}

Supplementary Data. All measured values from which the average values reported in the tables were calculated and details on the uncertainties for each measured point.

\section{Acknowledgements}

We gratefully acknowledge the generous donation of the spherical-resonator cell, which comprises the core of our instrument, to NIST in 2004 by Dr. J. T. R. Watson (1941-2006) of the National Engineering Laboratory (Glasgow, UK). We also gratefully acknowledge Prof. J. P. M. Trusler of Imperial College (London, UK) for designing and directing the fabrication of this spherical-resonator cell and for his guidance as we got it working. We thank Dr. M. R. Moldover of NIST for inspiration and enlightening guidance on the sound speed of gases and spherical 
resonators. We thank Ryan Hulse and Dr. Rajiv Singh of Honeywell for providing the highpurity sample of R1234ze(E). The measurements on RE347mcc were supported by a subcontract with United Technologies Research Center under DOE Agreement DE-EE0002770 (Tailored Working Fluids for Enhanced Geothermal Power Plants). This sponsor was not involved in the study design, nor the collection, analysis, and interpretation of the data. 


\section{References}

[1] M.R. Moldover, R.M. Gavioso, J.B. Mehl, L. Pitre, M. de Podesta, J.T. Zhang, Acoustic gas thermometry, Metrologia 51 (2014) R1-R19.

[2] M.R. Moldover, J.B. Mehl, M. Greenspan, Gas-filled spherical resonators: Theory and experiment, J. Acoust. Soc. Am. 79 (1986) 253-272.

[3] J.P.M. Trusler, M. Zarari, The speed of sound and derived thermodynamic properties of methane at temperatures between $275 \mathrm{~K}$ and $375 \mathrm{~K}$ and pressures up to $10 \mathrm{MPa}$, J. Chem. Thermodyn. 24 (1992) 973-991.

[4] Ø. Hodnebrog, M. Etminan, J.S. Fuglestvedt, G. Marston, G. Myhre, C.J. Nielsen, K.P. Shine, T.J. Wallington, Global warming potentials and radiative efficiencies of halocarbons and related compounds: A comprehensive review, Rev. Geophys. 51 (2013) 300-378.

[5] 3M, Product Information: 3M Novec 7000 Engineered Fluid, http://multimedia.3m.com/mws/media/121372O/3m-novec-7000-engineered-fluidtds.pdf?fn=Novec\%207000\%20Engineered\%20Fluid\%20TDS_, 2014.

[6] ASHRAE, ANSI/ASHRAE Standard 34-2013 Designation and Safety Classification of Refrigerants, ASHRAE, Atlanta, GA, 2013.

[7] J.P.M. Trusler, Physical Acoustics and Metrology of Fluids, CRC Press, Boca Raton, FL, 1991.

[8] A.F. Estrada-Alexanders, J.P.M. Trusler, Speed of sound in carbon dioxide at temperatures between (220 and 450) $\mathrm{K}$ and pressures up to $14 \mathrm{MPa}$, J. Chem. Thermodyn. 30 (1998) 1589-1601.

[9] F.O. Goodman, Thermal accomodation coefficients, J. Phys. Chem. 84 (1980) 1431-1445.

[10] D.J. Rader, W.M. Trott, J.R. Torczynski, J.N. Castaneda, T.W. Grasser, Measurements of Thermal Accommodation Coefficients, Sandia National Laboratories, Albuquerque, New Mexico, 2005.

[11] J.D. Lambert, Vibrational and rotational relaxation in gases, Oxford University Press, Oxford, 1977.

[12] Y. Zhou, E.W. Lemmon, Equations of state for RE345cb2, RE347mcc, RE245fa2, and R1216, J. Phys. Chem. Ref. Data (In Preparation) (2015).

[13] M. Thol, E.W. Lemmon, Equation of State for the Thermodynamic Properties of trans1,3,3,3-Tetrafluoropropene [R1234ze(E)], Int. J. Thermophys. (In Preparation) (2015). 
[14] M.B. Ewing, J.P.M. Trusler, On the analysis of acoustic resonance measurement, J. Acoust. Soc. Am. 85 (1989) 1780-1782.

[15] J.G. Hust, J. Filla, D.R. Smith, A modified digital PID temperature controller for thermal property measurements, J. Therm. Insulat. 11 (1987) 102-107.

[16] T.J. Bruno, P.D.N. Svoronos, CRC Handbook of Basic Tables for Chemical Analysis, 3rd ed., Taylor and Francis, CRC Press, Boca Raton, FL, 2011.

[17] T.J. Bruno, P.D.N. Svoronos, CRC Handbook of Fundamental Spectroscopic Correlation Charts, Taylor and Francis, CRC Press, Boca Raton, FL, 2005.

[18] U. Setzmann, W. Wagner, A new equation of state and tables of thermodynamic properties for methane covering the range from the melting line to $625 \mathrm{~K}$ at pressures to 1000 MPa, J. Phys. Chem. Refer. Data 20 (1991) 1061-1151.

[19] D. Bücker, W. Wagner, A reference equation of state for the thermodynamic properties of ethane for temperatures from the melting line to $675 \mathrm{~K}$ and pressures up to $900 \mathrm{MPa}$, J. Phys. Chem. Refer. Data 35 (2006) 205-266.

[20] A.F. Estrada-Alexanders, J.P.M. Trusler, The speed of sound and derived thermodynamic properties of ethane at temperatures between $220 \mathrm{~K}$ and $450 \mathrm{~K}$ and pressures up to 10.5 MPa, J. Chem. Thermodyn. 29 (1997) 991-1015.

[21] M.B. Ewing, A.R.H. Goodwin, Speeds of sound, perfect-gas heat-capacities, and acoustic virial-coefficients for methane determined using a spherical resonator at temperatures between $255 \mathrm{~K}$ and $300 \mathrm{~K}$ and pressures in the range $171 \mathrm{kPa}$ to $7.1 \mathrm{kPa}$, J. Chem. Thermodyn. 24 (1992) 1257-1274.

[22] Y. Kano, Y. Kayukawa, K. Fujii, Ideal gas heat capacity derived from speed of sound measurements in the gaseous phase for trans-1,3,3,3-tetrafluoropropene, J. Chem. Eng. Data 58 (2013) 2966-2969.

[23] P.J. Mohr, B.N. Taylor, D.B. Newell, CODATA recommended values of the fundamental physical constants: 2010, Rev. Mod. Phys. 84 (2012) 1527-1605.

[24] C. Tegeler, R. Span, W. Wagner, A new equation of state for argon covering the fluid region for temperatures from the melting line to $700 \mathrm{~K}$ at pressures up to $1000 \mathrm{MPa}, \mathrm{J}$. Phys. Chem. Refer. Data 28 (1999) 779-850.

[25] A.F. Estrada-Alexanders, J.P.M. Trusler, The speed of sound in gaseous argon at temperatures between $110 \mathrm{~K}$ and $450 \mathrm{~K}$ and at pressures up to $19 \mathrm{MPa}$, J. Chem. Thermodyn. 27 (1995) 1075-1089.

[26] M.B. Ewing, A.R.H. Goodwin, An apparatus based on a spherical resonator for measuring the speed of sound in gases at high pressures - results for argon at temperatures in the 
range $255 \mathrm{~K}$ to $300 \mathrm{~K}$ and pressures up to $7 \mathrm{MPa}$, J. Chem. Thermodyn. 24 (1992) 531547.

[27] S.J. Boyes, PhD. Thesis, The speed of sound in gases with application to equations of state and sonic nozzles, University College, London, 1992.

[28] M. Zarari, PhD. Thesis, Intermolecular forces from the speed of sound in gases, Department of Chemical Engineering and Chemical Technology, Imperial College, London, 1993.

[29] R.C. Paule, J. Mandel, Consensus values and weighting factors, J. Res. Natl. Bur. Standards 87 (1982) 377-385. 
TABLE 1. Sample information.

\begin{tabular}{|c|c|c|c|c|c|}
\hline $\begin{array}{l}\text { Chemical } \\
\text { Name }\end{array}$ & Source & $\begin{array}{l}\text { Initial Purity/ } \\
\text { mole frac. }\end{array}$ & $\begin{array}{l}\text { Purification } \\
\text { Method }\end{array}$ & $\begin{array}{l}\text { Final Purity/ } \\
\text { mole frac. }\end{array}$ & $\begin{array}{l}\text { Analysis } \\
\text { Method }\end{array}$ \\
\hline argon & Matheson & 0.999999 & none & & \\
\hline methane & Matheson & 0.99999 & none & 0.99999 & $\mathrm{GC} \mathrm{MS}^{\mathrm{a}}$ \\
\hline ethane & Matheson & 0.9999 & none & 0.9999 & $\mathrm{GC} / \mathrm{MS}^{\mathrm{a}}$ \\
\hline RE347mcc ${ }^{\mathrm{b}}$ & $3 \mathrm{M}$ & 0.9989 & degassing & 0.9989 & $\mathrm{GC} / \mathrm{MS}^{\mathrm{a}}$ \\
\hline $\mathrm{R} 1234 \mathrm{ze}(\mathrm{E})^{\mathrm{c}}$ & Honeywell & 0.99993 & degassing & 0.99993 & GC/MS/IR \\
\hline
\end{tabular}

${ }^{\mathrm{a}}$ Gas chromatography/mass spectrometry

${ }^{b} 1,1,1,2,2,3,3$-heptafluoro-3-methoxypropane

${ }^{c}$ trans-1,3,3,3-tetrafluoropropene

${ }^{\mathrm{d}}$ Gas chromatography/mass spectrometry/infrared spectroscopy 
TABLE 2. Summary of errors and resulting uncertainty in the speed of sound.

\begin{tabular}{|c|c|c|c|c|c|}
\hline \multirow{2}{*}{ Source } & \multirow{2}{*}{ Magnitude of error } & \multicolumn{4}{|c|}{ Range of uncertainties in sound speed $/\left(\mathrm{m} \cdot \mathrm{s}^{-1}\right.$, unless indicated $)$} \\
\hline & & methane & ethane & RE347mcc & R1234ze(E) \\
\hline \multicolumn{6}{|c|}{ State-point uncertainties } \\
\hline Temperature & $0.020 \mathrm{~K}$ & $0.0100-0.0148$ & $0.0075-0.0106$ & $0.0029-0.0091$ & $0.0042-0.0120$ \\
\hline Pressure & $\mathrm{a}$ & $<0.0001$ & $0.0001-0.0005$ & $0.0002-0.0161$ & $0.0002-0.0104$ \\
\hline Sample purity & $0.0003 \%-0.01 \%$ & $0.0013-0.0017$ & $0.0059-0.0077$ & $0.0091-0.0146$ & $0.0118-0.0180$ \\
\hline Sample instability & $5 \%$ of correction & $\sim 0$ & $\sim 0$ & $0-0.0186$ & $0-0.0013$ \\
\hline \multicolumn{6}{|l|}{ Sphere diameter } \\
\hline$D_{0}(T=300 \mathrm{~K})$ & $0.0040 \mathrm{~mm}$ & $0.0217-0.0276$ & $0.0147-0.0193$ & $0.0046-0.0073$ & $0.0059-0.0090$ \\
\hline$D(T)$ & $0.00004 \mathrm{~mm} \times(T / \mathrm{K}-300)$ & $0.0009-0.0048$ & $0.0004-0.0034$ & $0.0001-0.0015$ & $0.0000-0.0011$ \\
\hline$D(p)$ & $0.00016 \mathrm{~mm} \times p / \mathrm{MPa}$ & $0.0032-0.0054$ & $0.0026-0.0048$ & $0.0001-0.0048$ & $0.0002-0.0078$ \\
\hline$D(t)$ [stability] & $0.0013 \mathrm{~mm}$ & $0.0071-0.0090$ & $0.0048-0.0063$ & $0.0015-0.0024$ & $0.0019-0.0029$ \\
\hline \multicolumn{6}{|c|}{ Fitting of and corrections to resonance peak } \\
\hline Half-width & $1 \%$ of half-width & $0.0013-0.0056$ & $0.0002-0.0010$ & $0.0000-0.0012$ & $0.0001-0.0011$ \\
\hline Boundary layer & $1 \%-10 \%$ of correction & $0.0003-0.0006$ & $<0.0002$ & $<0.0002$ & $<0.0002$ \\
\hline Shell resonance & $10 \%$ of correction & $0.0001-0.0011$ & $<0.0002$ & $<0.0002$ & $<0.0002$ \\
\hline Dispersion & $5 \%$ of correction & $<0.0005$ & $<0.0001$ & $\sim 0$ & $\sim 0$ \\
\hline \multicolumn{6}{|c|}{ Combined and averaged uncertainties } \\
\hline \multicolumn{2}{|c|}{$u_{\mathrm{c}}$ for a given mode $(k=1)^{\mathrm{b}}$} & $0.0276-0.0321$ & $0.0199-0.0236$ & $0.0135-0.0212$ & $0.0171-0.0216$ \\
\hline \multicolumn{2}{|c|}{ standard deviation across modes } & $0.0065-0.0399$ & $0.0049-0.0128$ & $0.0005-0.0103$ & $0.0005-0.0183$ \\
\hline \multicolumn{2}{|c|}{$\begin{array}{l}\text { combined expanded }(k=2) \text { uncertainty } \\
\text { averaged across all modes }{ }^{\mathrm{b}}\end{array}$} & $0.0575-0.1001$ & $0.0423-0.0536$ & $0.0285-0.0427$ & $0.0469-0.0920$ \\
\hline \multicolumn{2}{|c|}{$\begin{array}{l}\text { combined expanded }(k=2) \text { uncertainty averaged } \\
\text { across all modes, including sample instability }\end{array}$} & $0.0575-0.1001$ & $0.0423-0.0536$ & $0.0285-0.0504$ & $0.0469-0.0920$ \\
\hline \multicolumn{2}{|c|}{$\begin{array}{l}\text { relative combined expanded }(k=2) \text { uncertainty } \\
\text { averaged across all modes, including sample } \\
\text { instability }\end{array}$} & $(0.012-0.020) \%$ & $(0.013-0.015) \%$ & $(0.023-0.045) \%$ & $(0.034-0.054) \%$ \\
\hline
\end{tabular}




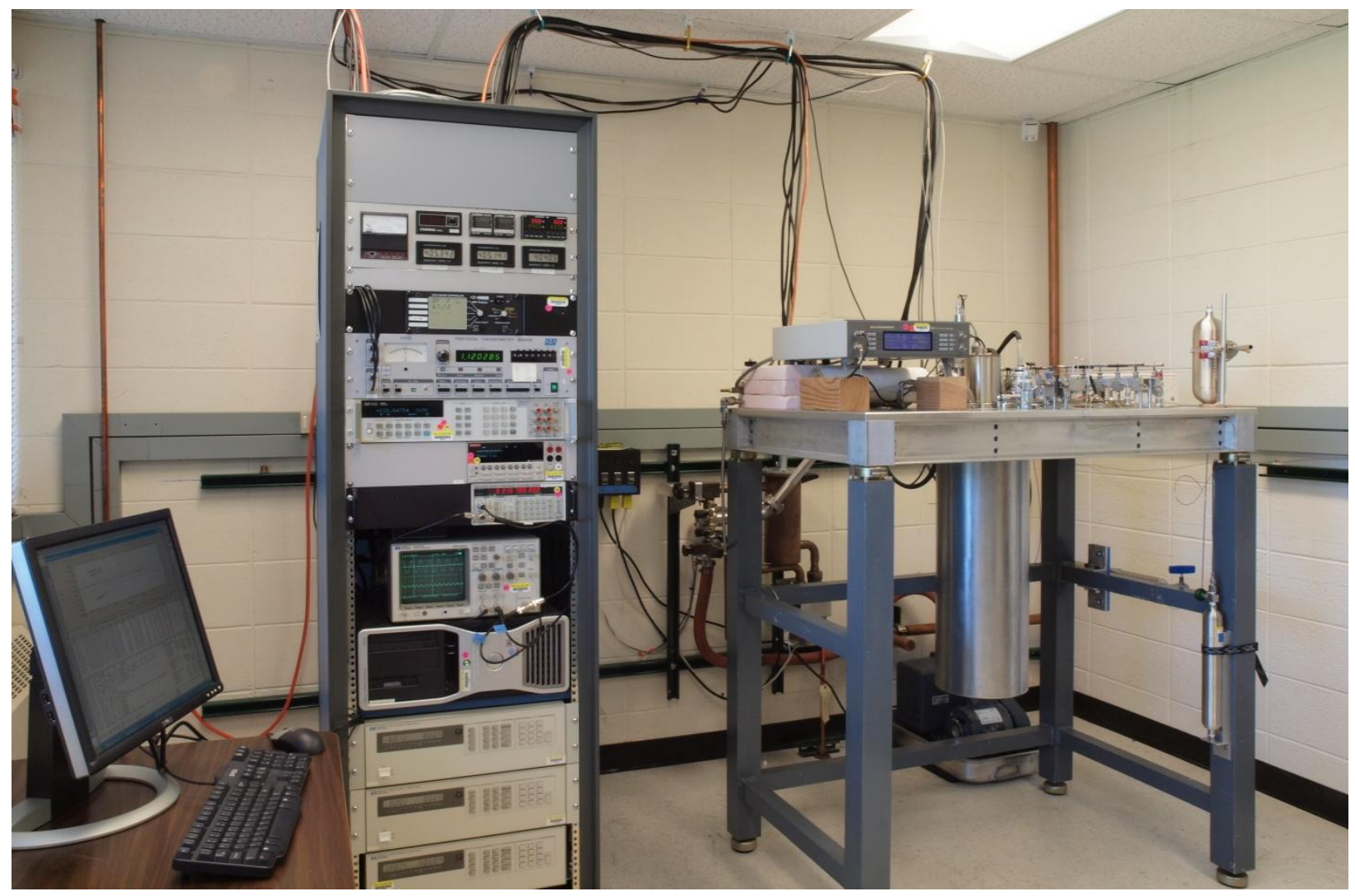

FIGURE 1. Photo of the complete sound-speed apparatus. 
FIGURE 4. Details of the dielectric-membrane transducers to excite and measure the acoustic resonance of the gas within the sphere that is typically at elevated pressures of up to $40 \mathrm{MPa}$. 


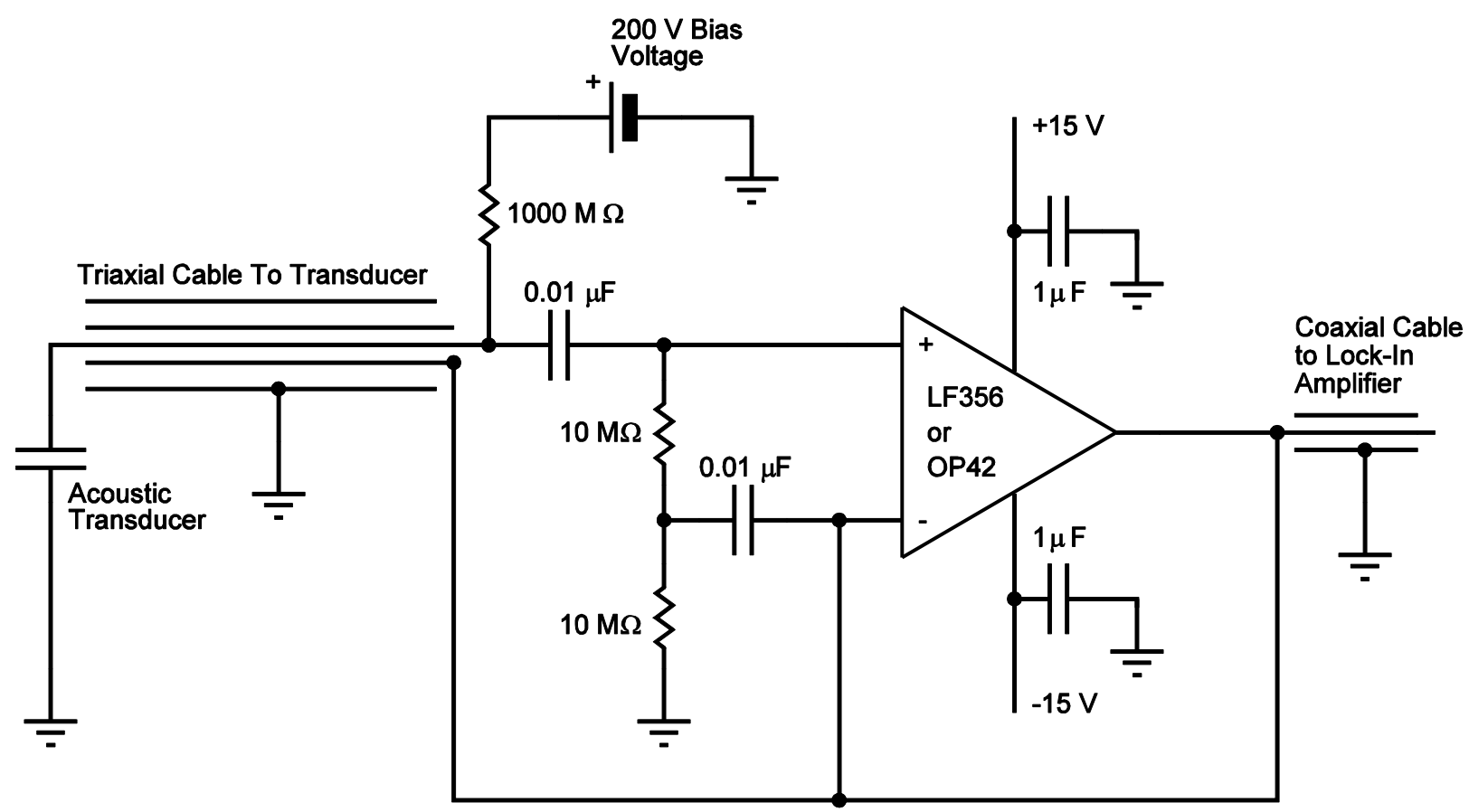

FIGURE 5. Schematic diagram of the bootstrapped JFET operational amplifier circuit with unity gain that drives the intermediate shield of the triaxial cable connecting the dielectric acoustic transducer to the amplifier input and which provides the signal (through coaxial cable) to the lock-in amplifier. A DC bias voltage of up to $200 \mathrm{~V}$ is applied to the transducer membrane and isolated from the amplifier with the input capacitor. 


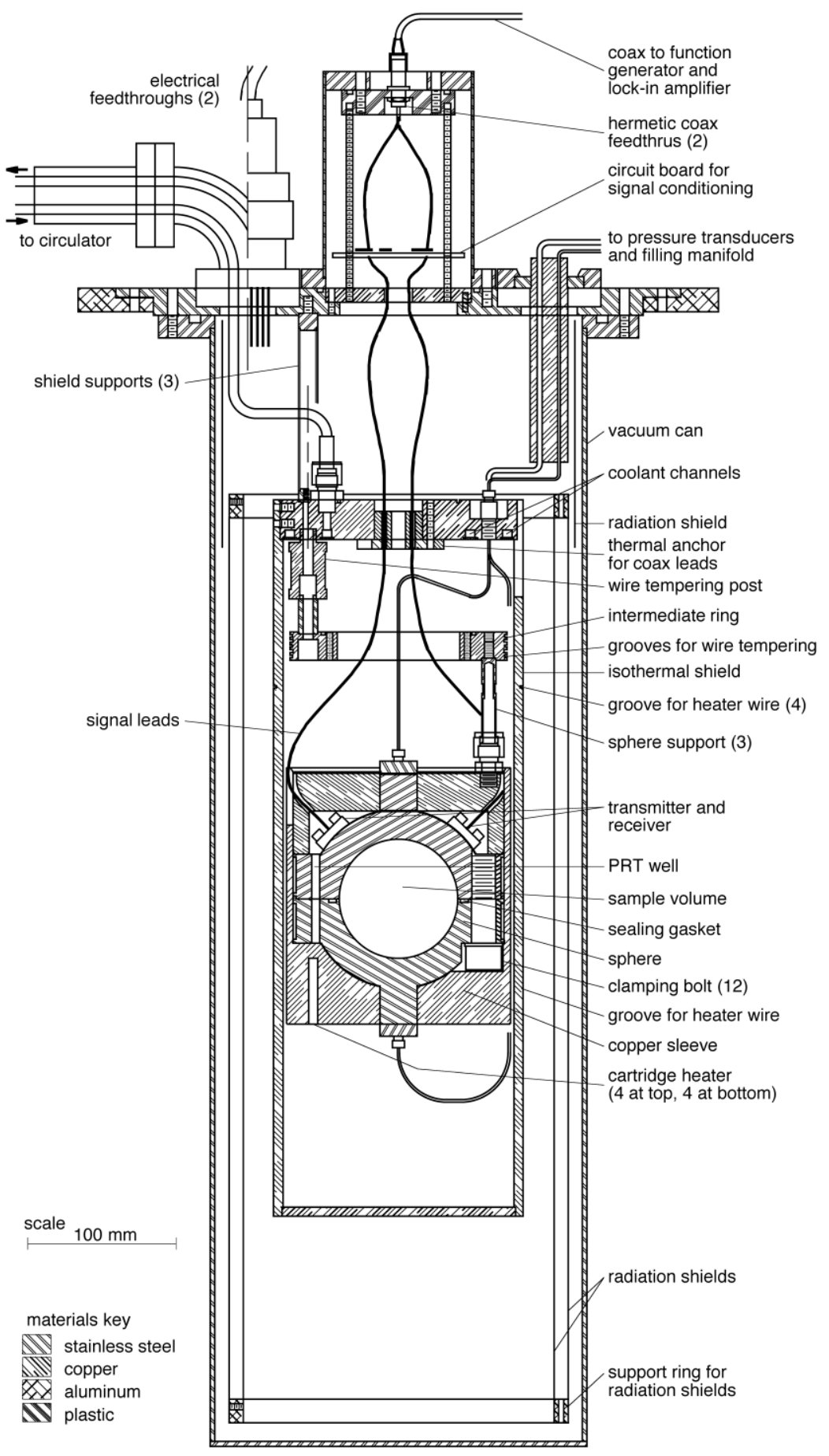

FIGURE 6. Schematic diagram of the speed of sound thermostat. 
FIGURE 7. Photo of the resonator sphere and the four parts of the copper sleeve that reduces temperature gradients. The two bottom parts are removed in the picture but are firmly attached with good thermal contact, enclosing the sphere during operation. 
FIGURE 8. Photo of spherical resonator with isothermal block, intermediate tempering ring and isothermal shield support. The isothermal shield and radiation shield layers enclose this entire assembly during operation. All these parts are in turn enclosed in the vacuum can, which is evacuated to provide excellent thermal insulation. 


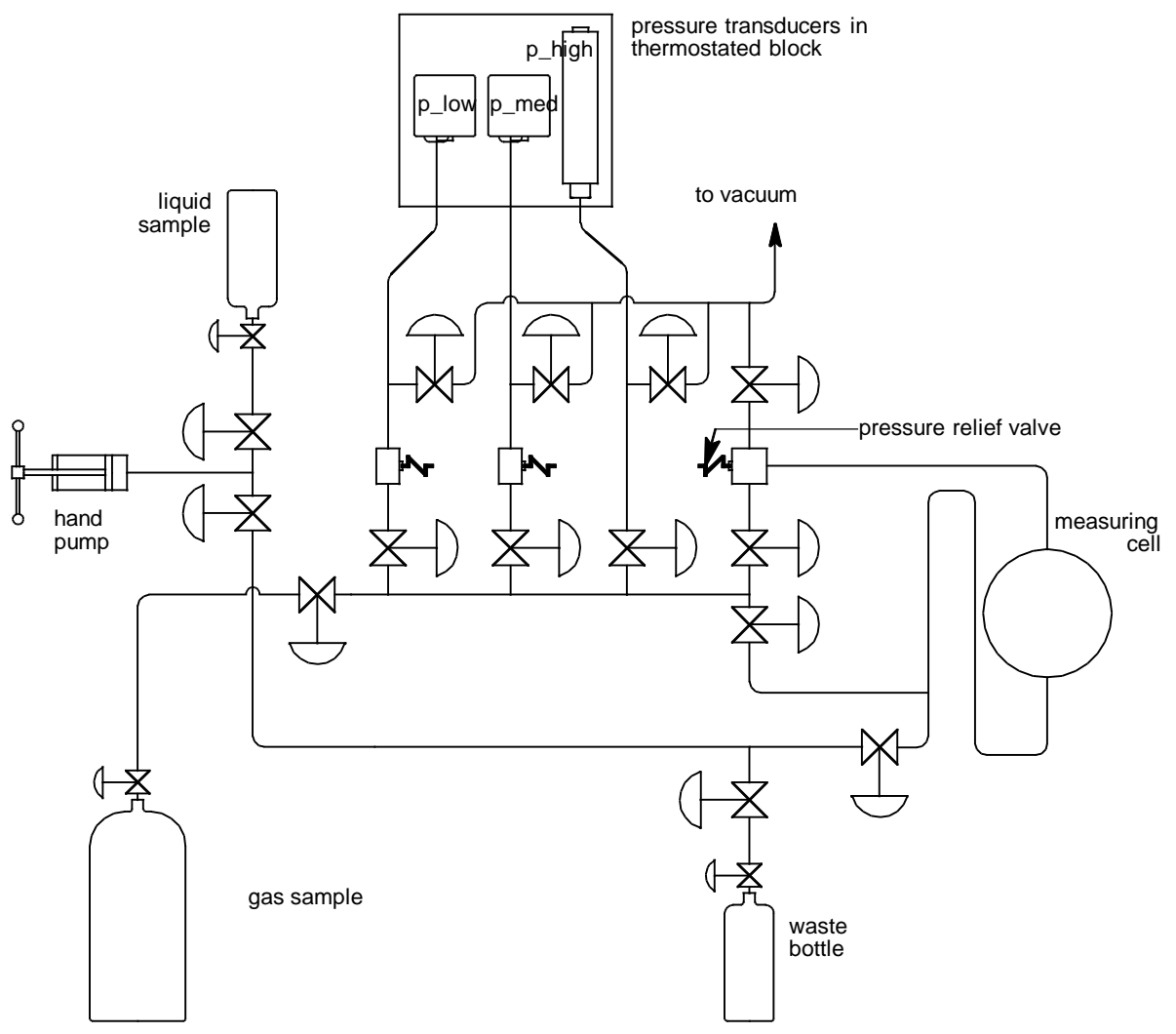

FIGURE 9. High-pressure sample-handling manifold for spherical resonator. 


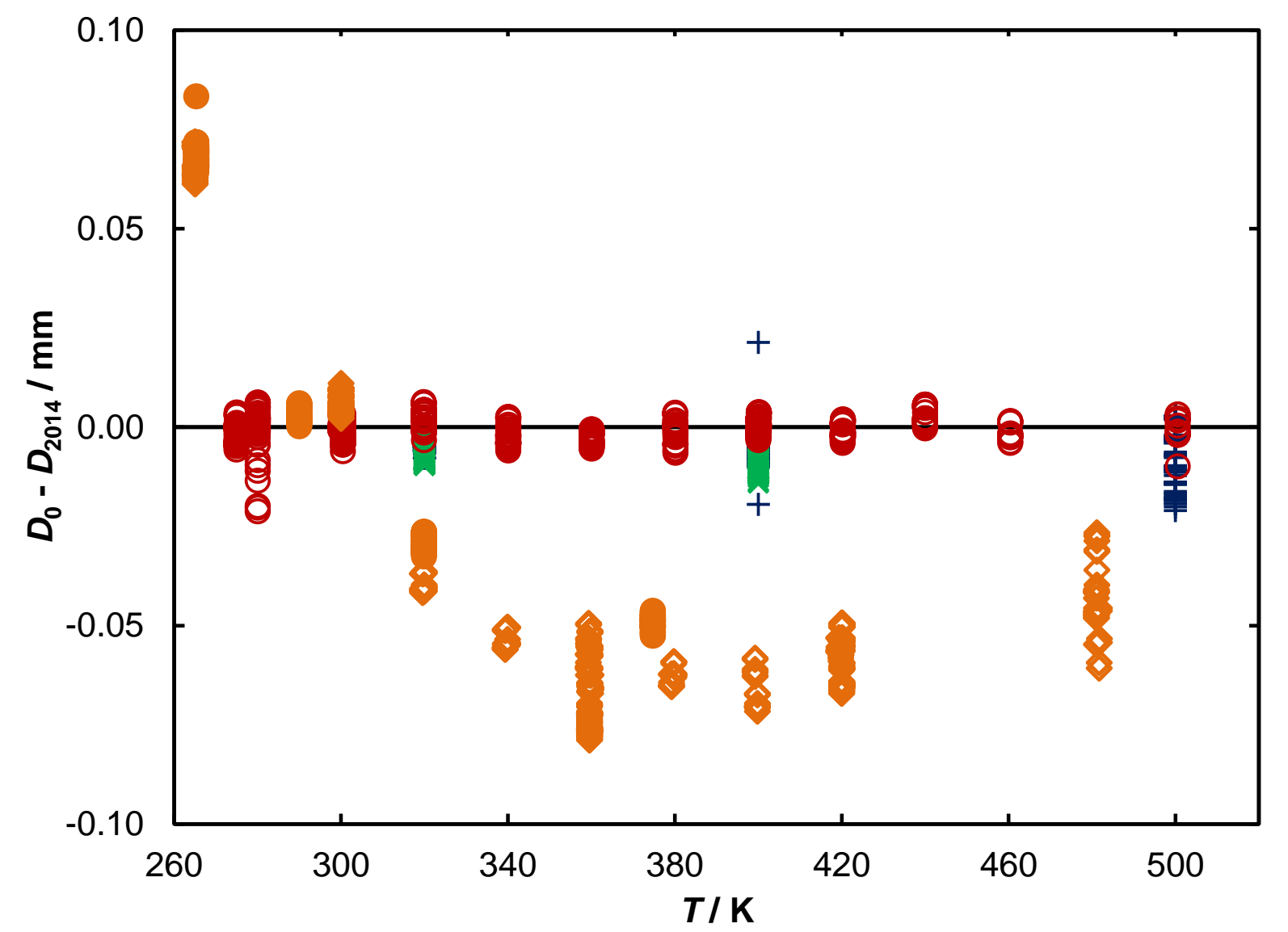

FIGURE 10. Deviations between the calibration measurements of the sphere diameter at zero pressure from the calibration function of equation 12 for April 2014 data. Calibration series designated: 1) + , November to December 2011; 2) $\times$, March 2012; 3) $\diamond$, August to September 2012; 4) O, April 2014. Since the nominal inner diameter of the sphere is $80 \mathrm{~mm}$, differences of $0.05 \mathrm{~mm}$ in diameter correspond to relative fractional differences of 0.000625 . 
FIGURE 11. Deviations between the sound speed measured for a) methane and $b$ ) ethane and the equations of state of Setzmann and Wagner [18] and Bücker and Wagner [19] respectively. The deviations are designated by: $O$, methane March 2013; $\mathbf{x}$, methane July 2014; $\triangle$, Trusler and Zarari methane [3]; $\diamond$, Ewing and Goodwin [21] methane ( $p \leq 2 \mathrm{MPa}$ ); $\square$, ethane March 2013; + , ethane July 2014; and $\triangle$, Estrada-Alexanders and Trusler ethane [20] ( $p \leq 2 \mathrm{MPa})$. 


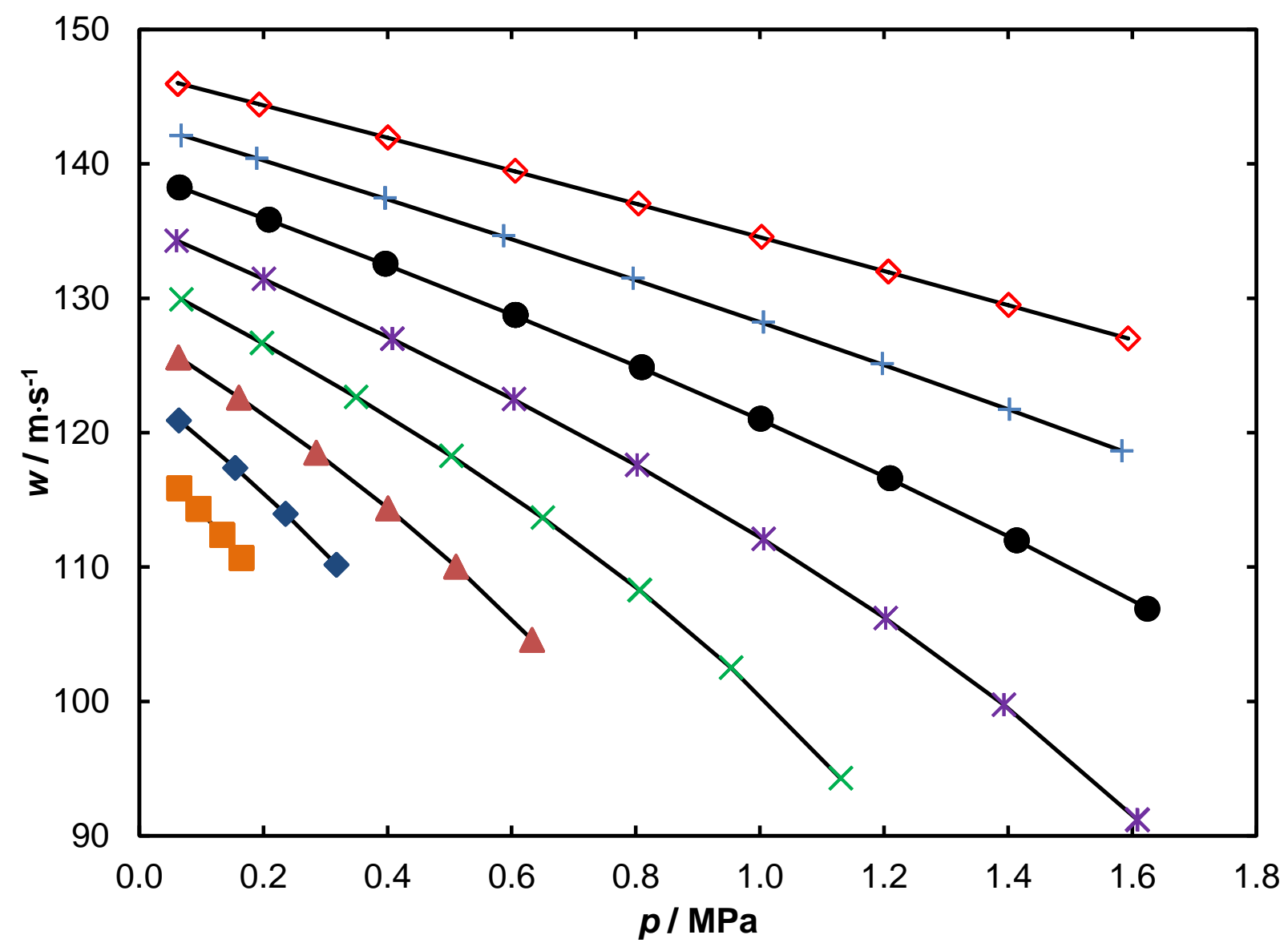

FIGURE 12. Sound speed of RE347mcc at temperatures from $325 \mathrm{~K}$ to $500 \mathrm{~K}$ with lines calculated with the equation of state of Zhou and Lemmon.[12] The isotherm temperatures are designated by: $\mathbf{\square}, 325 \mathrm{~K} ; \diamond, 350 \mathrm{~K} ; \boldsymbol{\Delta}, 375 \mathrm{~K} ; \mathbf{x}, 400 \mathrm{~K} ; *, 425 \mathrm{~K} ; \bullet, 450 \mathrm{~K} ;+$, $475 \mathrm{~K} ; \diamond$, $500 \mathrm{~K}$. 


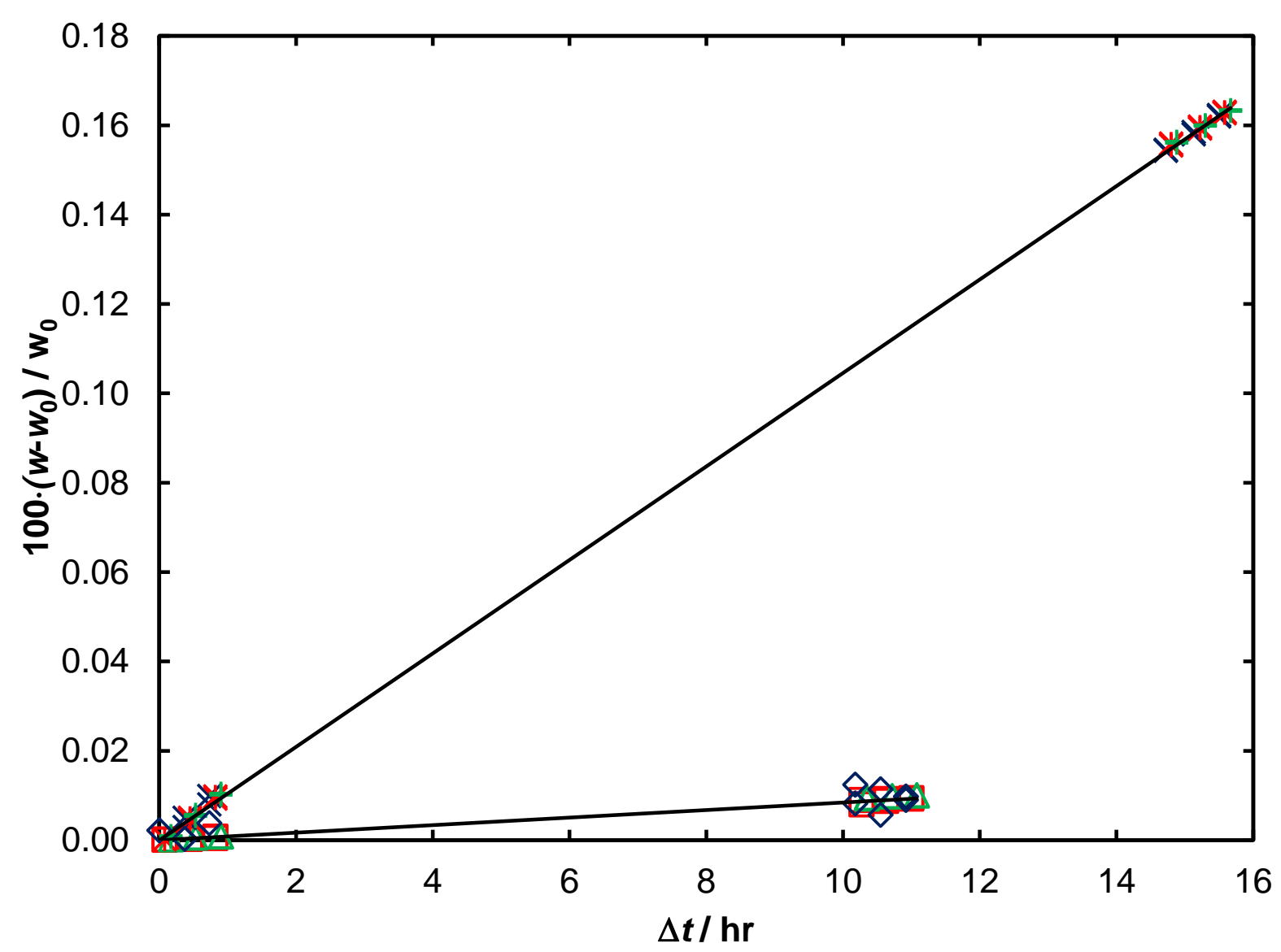

FIGURE 13. Stability of the sound speed of RE347mcc as a function of residence time at $500 \mathrm{~K}$ and pressures of $62 \mathrm{kPa}$ and $400 \mathrm{kPa}$. The rate of increase in measured sound speed decreases with pressure (gas inventory in cell). The pressures and modes are designated by: $\diamond, 400 \mathrm{kPa}$ $(0,2) ; \square, 400 \mathrm{kPa}(0,3) ; \triangle, 400 \mathrm{kPa}(0,4) ; \mathbf{x}, 62 \mathrm{kPa}(0,2) ; *, 62 \mathrm{kPa}(0,3) ;+, 62 \mathrm{kPa}(0,4)$. 


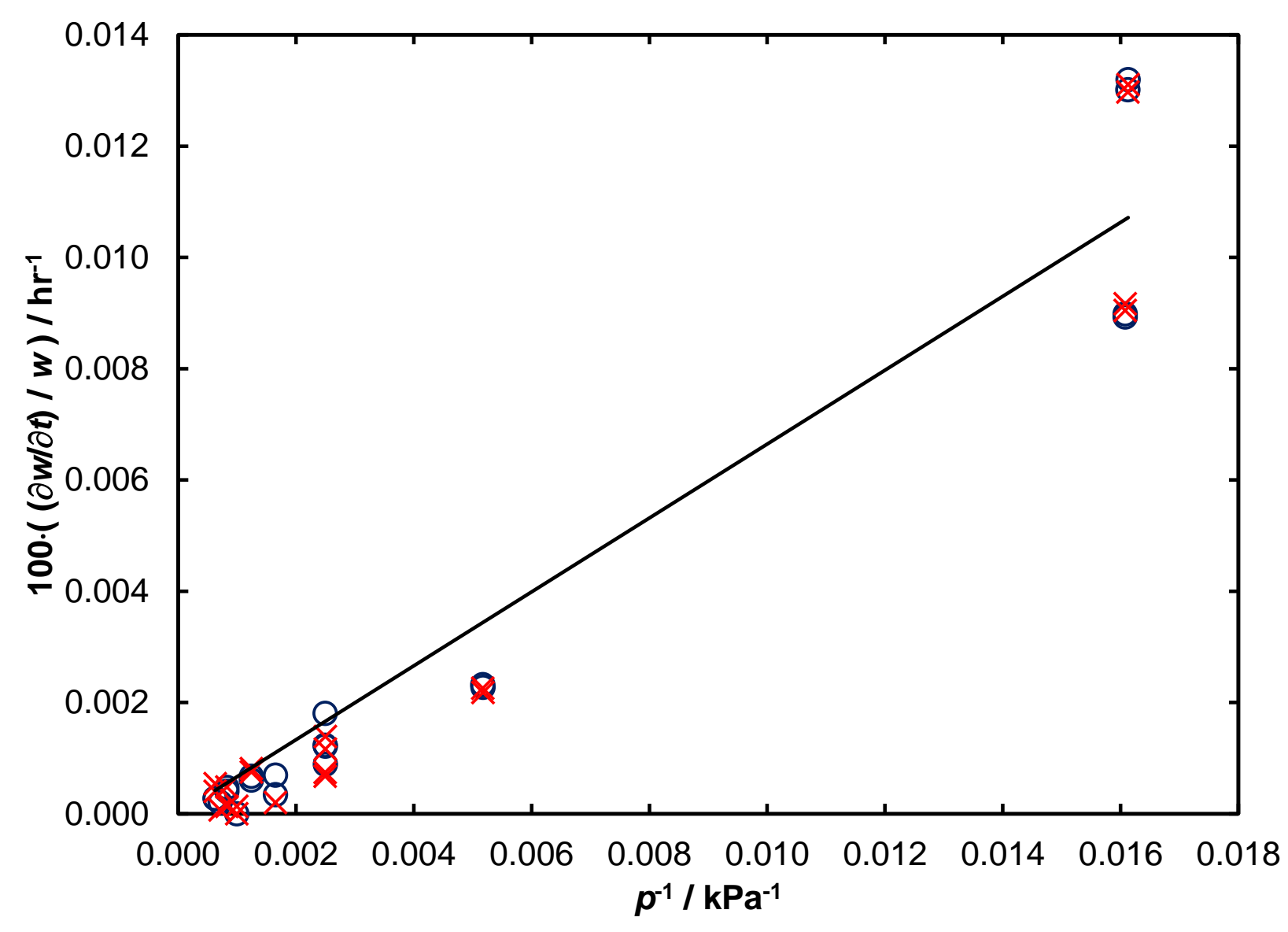

FIGURE 14. The pressure dependence of the rate of change in measured sound speed $a(T)$ for $\mathrm{RE} 347 \mathrm{mcc}$ at $500 \mathrm{~K}$ as a function of pressure in $\mathrm{kPa}$. Spherical resonance modes are designated as $\mathbf{X},(0,3) ; \mathrm{O},(0,4)$. 


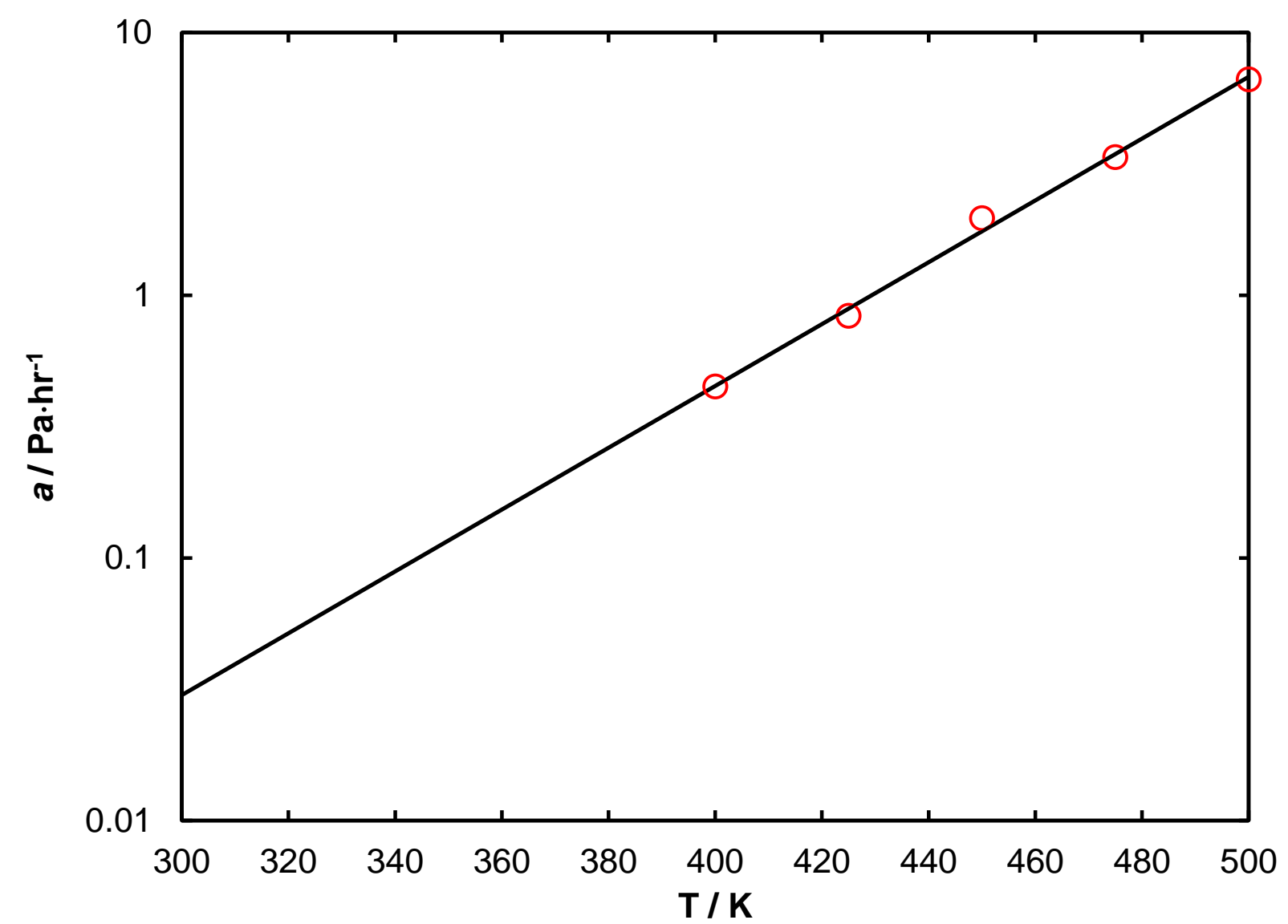

FIGURE 15. The exponential temperature dependence of the rate of change in sound speed coefficient $a(T)$ for RE347mcc. 


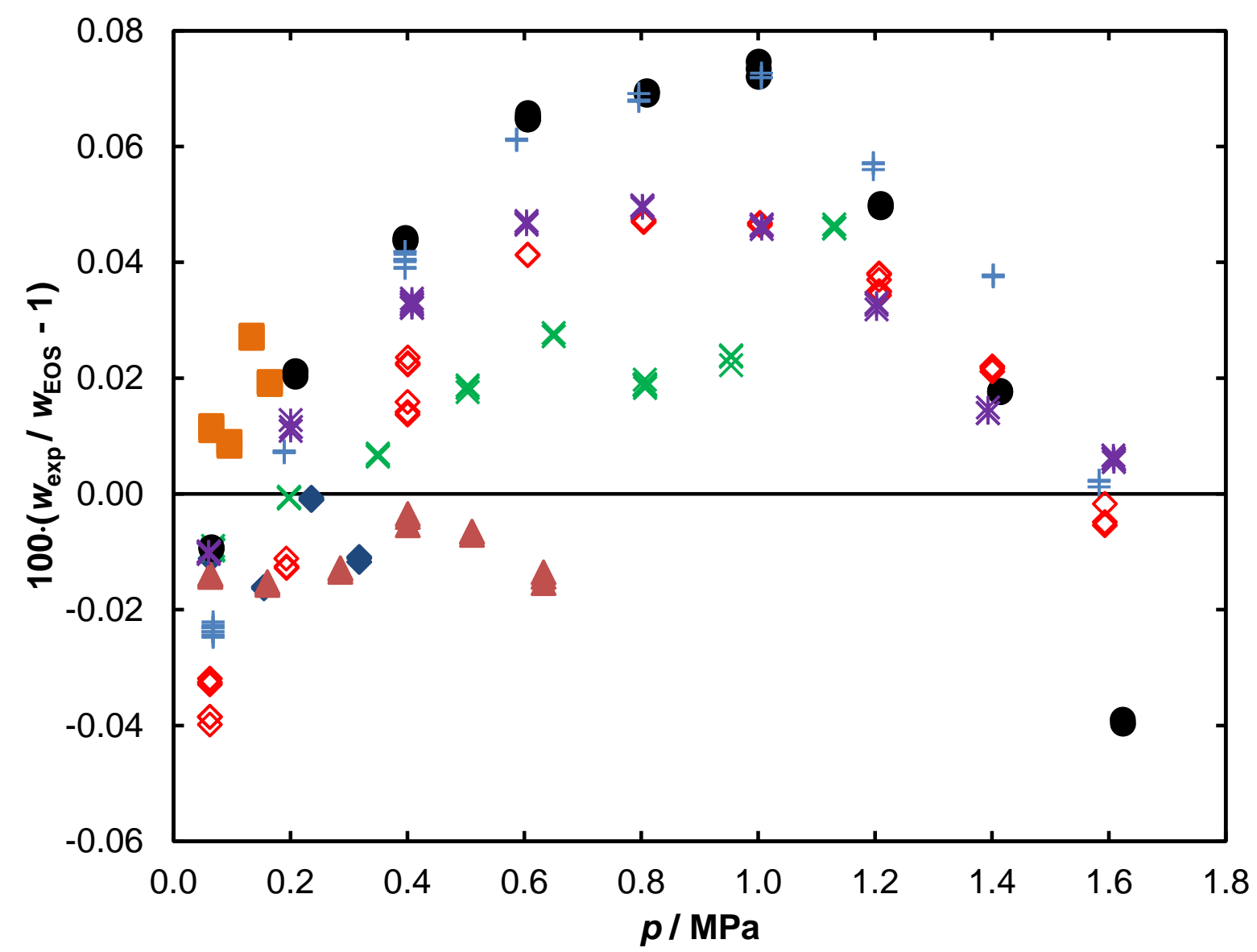

FIGURE 16. Deviations between the measured sound speed of RE347mcc and the equation of state of Zhou and Lemmon.[12] The isotherm temperatures are designated by: $\mathbf{\square}, 325 \mathrm{~K} ; \diamond, 350$ $\mathrm{K} ; \boldsymbol{\Lambda}, 375 \mathrm{~K} ; \mathbf{x}, 400 \mathrm{~K} ; *, 425 \mathrm{~K} ; \bullet, 450 \mathrm{~K} ;+, 475 \mathrm{~K} ; \diamond, 500 \mathrm{~K}$. 


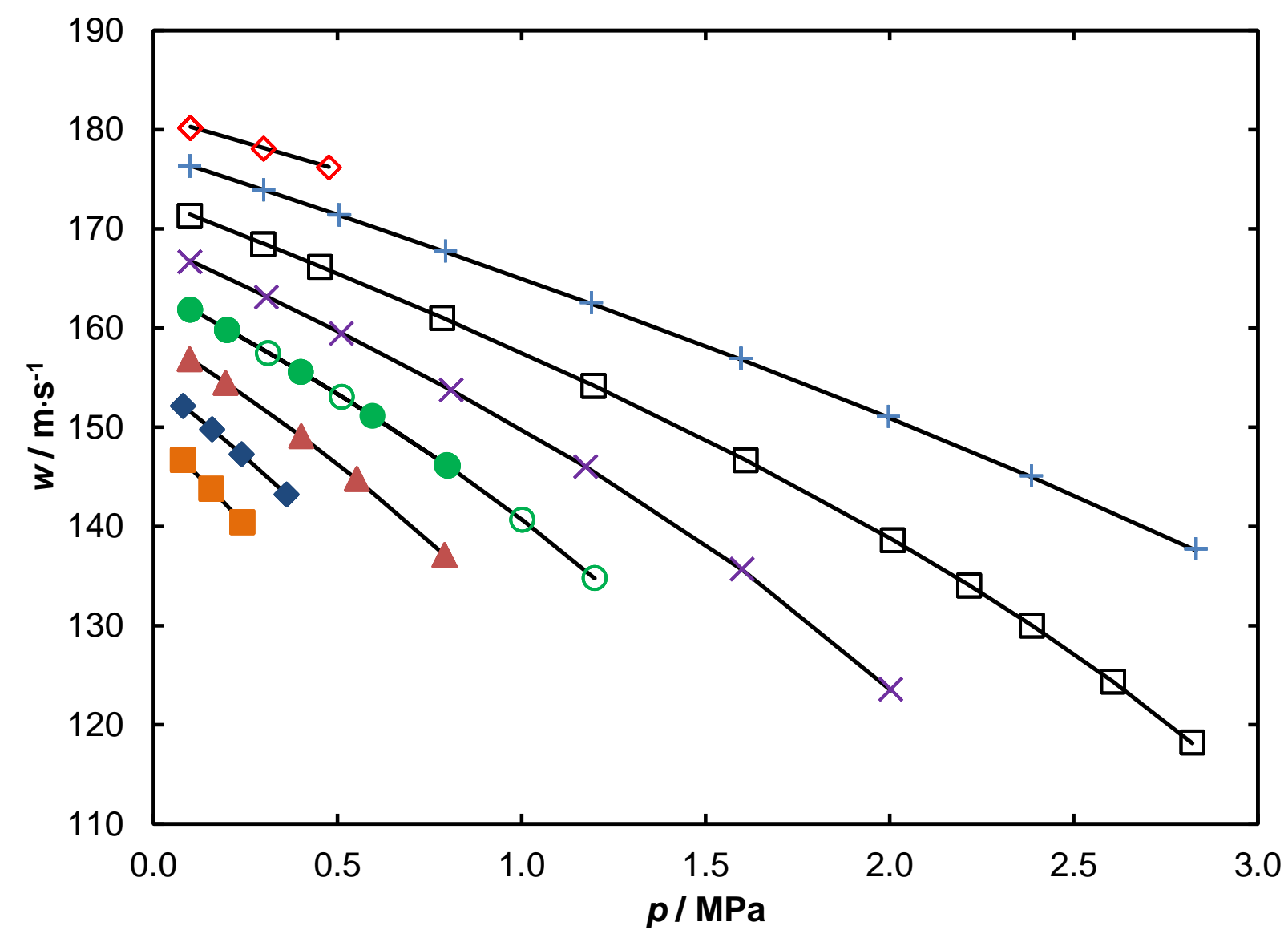

FIGURE 17. Sound speed of R1234ze(E) measured along isotherms at temperatures from $280 \mathrm{~K}$ to $420 \mathrm{~K}$ is shown along with the values from the equation of state of Thol and Lemmon.[13] The isotherm temperatures are designated by: $\boldsymbol{\square}, 280 \mathrm{~K} ; \diamond, 300 \mathrm{~K} ; \boldsymbol{\Delta}, 320 \mathrm{~K} ; \boldsymbol{\bullet}, 340 \mathrm{~K}$ (a); ○, $340 \mathrm{~K}(\mathrm{~b}) ; \times \mathbf{x}, 360 \mathrm{~K} ; \square, 380 \mathrm{~K} ;+$, $400 \mathrm{~K} ; \diamond, 420 \mathrm{~K}$. 


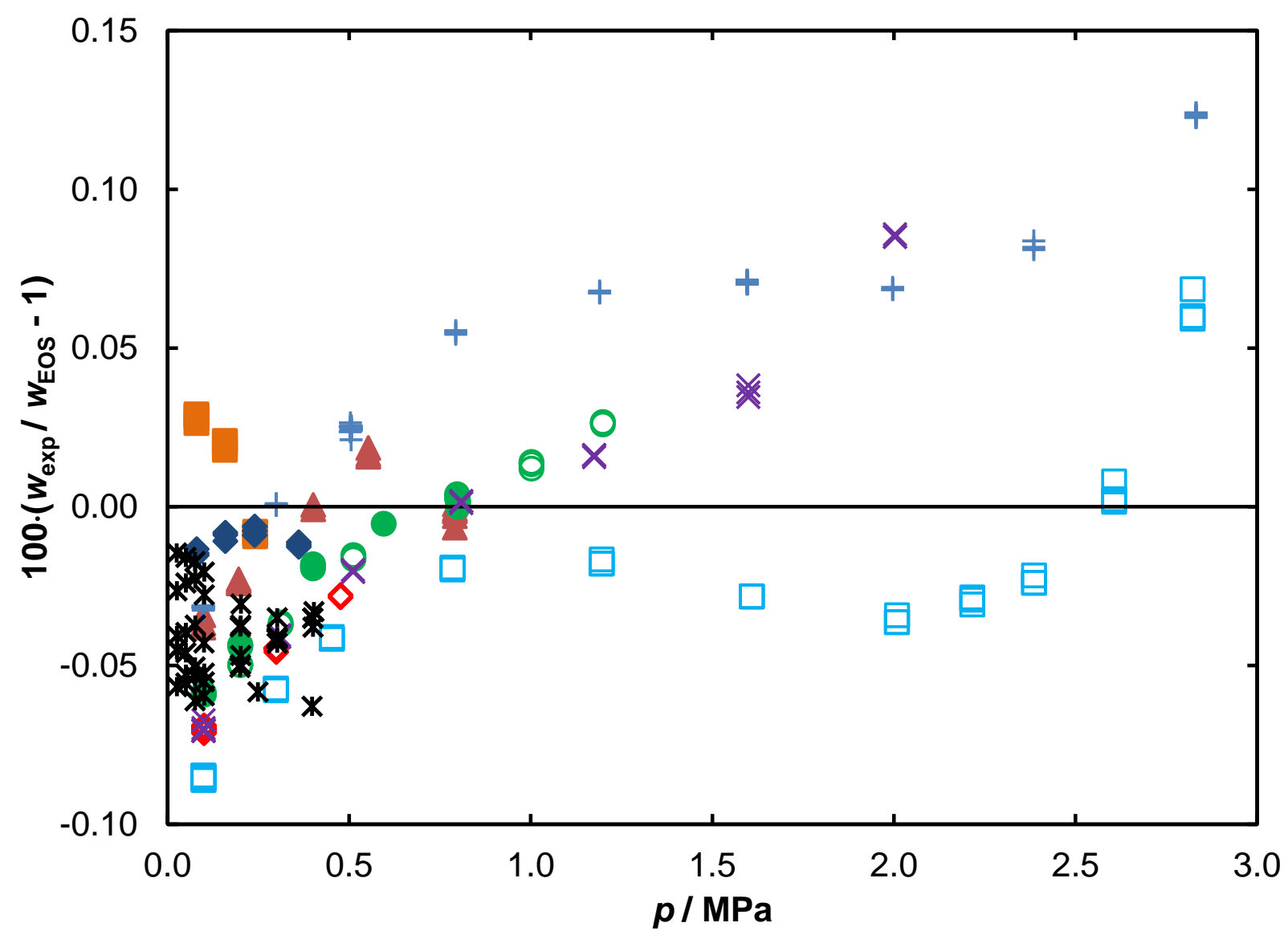

FIGURE 18. Deviations between the measured sound speed of R1234ze(E) and the values from the equation of state of Thol and Lemmon.[13] The isotherm temperatures are designated by: $\mathbf{\square}$, $280 \mathrm{~K} ; \diamond, 300 \mathrm{~K} ; \boldsymbol{\Delta}, 320 \mathrm{~K} ; \bullet, 340 \mathrm{~K}(\mathrm{a}) ;$ O, $340 \mathrm{~K}$ (b); $\boldsymbol{x}, 360 \mathrm{~K} ;$ ㅁ, $380 \mathrm{~K} ;+, 400 \mathrm{~K} ; \diamond$, $420 \mathrm{~K}$. The data of Kano et al. [22] for the sound speed of vapor R1234ze(E) at temperatures from $278 \mathrm{~K}$ to $353 \mathrm{~K}$ are designated by $*$. 